\title{
A flora e a vegetação dos afloramentos rochosos em três municípios na região Norte do Ceará, Brasil: caracterização fitossociológica
}

The flora and vegetation of rocky outcrops in three municipalities in the northern region of Ceará, Brazil: phytosociological characterization

\author{
Marízia Menezes Dias Pereira ${ }^{1,3,5}$, Petrônio Emanuel Timbó Braga ${ }^{2}$, Nuno Guiomar ${ }^{3}$, \\ Francisco Diego Sousa Santos ${ }^{2} \&$ Sílvia Ribeiro ${ }^{3,4}$
}

\begin{abstract}
Resumo
O presente estudo teve como objetivo identificar a flora e a vegetação dos afloramentos rochosos isolados e de baixa altitude (lajedos), na vegetação de Caatinga Arbustiva Aberta, que se encontram nos municípios de Sobral, Groaíras e Santa Quitéria, no estado do Ceará, Brasil e propor uma classificação fitossociológica para estas comunidades xerófilas. Foram definidas cinco áreas de coleta de dados com elevada proporção de rochas expostas (> 80\%) onde as excursões de campo decorreram em março de 2014 e 2015 ( $3^{\circ} 56^{\prime}$ 'S e 40 $20^{\circ}$ 'W, $4^{\circ} 01^{\prime} \mathrm{S}$ e 40 $05^{\prime} \mathrm{W}, 4^{\circ} 07^{\prime} \mathrm{S}$ e $40^{\circ} 08^{\prime} \mathrm{W}, 4^{\circ} 09^{\prime} \mathrm{S}$ e $40^{\circ} 09^{\prime} \mathrm{W}$ e $4^{\circ} 03^{\prime} \mathrm{S}$ e $40^{\circ} 00^{\prime} \mathrm{W}$ ). No estudo da vegetação aplicou-se os métodos TWINSPAN (two-way indicator species analysis) e o clássico sigmatista de Braun-Blanquet. As áreas mínimas dos inventários fitossociológicos variaram de 8 a $16 \mathrm{~m}^{2}$. Foram coletadas as espécies vegetais que crescem em fissuras, fendas e ilhas de vegetação que se encontram em afloramentos rochosos. Foram registradas 88 espécies, distribuídas em 59 gêneros e 30 famílias botânicas. Fabaceae foi a família que se destacou em riqueza específica (20 spp.), seguida por Poaceae (dez spp.), Euphorbiaceae (sete spp.) e Convolvulaceae (seis spp.). Quanto ao endemismo foram registradas, em vegetação rupestre, 19 espécies endêmicas para o Brasil. Na análise fitossociológica da vegetação propôs-se estudar a comunidade de Pilosocereus gounellei (FA.C.Weber) Byles \& Rowley e Encholirium spectabile Mart. ex Schult. \& Schult.f. e a de Crateva tapia L. e Combretum leprosum Mart. Palavras-chave: semiárido, elenco florístico, Caatinga, fitossociologia.
\end{abstract}

\begin{abstract}
In this study, we aim to identify flora and vegetation of low-elevation rocky outcrops (lajedos) in "Caatinga Arbustiva Aberta" (open shrublands) to propose a phytosociological classification for xerophilous communities. The study areas are located at the municipalities of Sobral, Groaíaras and Santa Quitéria (Ceará state, Brazil). We selected five stations comprising areas with high proportion of bare rocks ( $>80 \%)$, and the field work were conducted in March 2014 and 2015 respectively ( $3^{\circ} 56^{\prime} \mathrm{S}$ and $40^{\circ} 23^{\prime} \mathrm{W}, 4^{\circ} 01^{\prime} \mathrm{S}$ and $40^{\circ} 05^{\prime} \mathrm{W}, 4^{\circ} 07^{\prime} \mathrm{S}$ and $40^{\circ} 08^{\prime} \mathrm{W}$, $4^{\circ} 09^{\prime} \mathrm{S}$ and $40^{\circ} 09^{\prime} \mathrm{W}$ and $4^{\circ} 03^{\prime} \mathrm{S}$ and $40^{\circ} 00^{\prime} \mathrm{W}$ ). We have applied the TWINSPAN (two-way indicator species analysis) and Braun-Blanquet methods. The minimum areas of phytosociological inventories ranged from 8 to 16 $\mathrm{m}^{2}$. Floristic relevés were made and all the plant species growing in cracks, crevices and small vegetation "islands" were identified. The floristic list is composed of 88 species, distributed in 59 genera and 30 families. Fabaceae was the most representative in species richness, 20 species, followed by Poaceae (10 spp.), Euphorbiaceae ( 7 spp.) and Convolvulaceae (6 spp.). We identified 19 Brazilian endemisms. Following phytosociological analysis of vegetation we found two different communities: Pilosocereus gounellei FA.C.Weber) Byles \& Rowley and Encholirium spectabile Mart. ex Schult. \& Schult.f. and the Crateva tapia L. and Combretum leprosum Mart. Key words: semiarid, floristic list, xerophytes, Caatinga, phytosociology.
\end{abstract}

Veja material suplementar em <https://doi.org/10.6084/m9.figshare.5915233.v2>

\footnotetext{
${ }^{1}$ Universidade de Évora, Escola de Ciências e Tecnologia, Depto. Paisagem, Ambiente e Ordenamento, Colégio Luís António Verney, R. Romão Ramalho 59, Évora, Portugal.

${ }^{2}$ Universidade Estadual Vale do Acaraú (UVA), Curso de Ciências Biológicas, Av. da Universidade 850, Campus da Betânia, 62040-370, Sobral, CE, Brasil.

${ }^{3}$ Universidade de Évora, ICAAM - Instituto de Ciências Agrárias e Ambientais Mediterrânicas, Núcleo da Mitra, Apt. 94, 7002-554, Évora, Portugal.

${ }^{4}$ Universidade de Lisboa, Centro de Investigação em Agronomia, Alimentos, Ambiente e Paisagem (LEAF - Linking Landscape, Environment, Agriculture and Food), Instituto Superior de Agronomia, Tapada da Ajuda, 1349-017, Lisboa, Portugal.

${ }^{5}$ Autor para correspondência: mariziacmdp3@gmail.com
} 


\section{Introdução}

A Caatinga é um bioma endêmico do Brasil. Está localizado no semiárido brasileiro e ocupa uma área de $844.453 \mathrm{~km}^{2}$ (IBGE 2004). Sua vegetação é caracterizada pela elevada diversidade de espécies com grande variedade fisionômica no que se refere à quantidade e ao porte das espécies vegetais (Araújo et al. 2005; Costa et al. 2007; Apgaua et al. 2014; Pereira et al. 2014; Soares-Neto et al. 2014). A variação na estrutura e composição das comunidades vegetais está relacionada com os gradientes ambientais, tais como a topografia, a precipitação, teor de nutrientes e umidade edáfica (Ferraz et al. 1998; Tabarelli et al. 2003; Araújo et al. 2011). A geologia e o tipo de solos contribuem igualmente para a elevada heterogeneidade espacial evidenciada na distribuição da vegetação na escala local, mesmo que em áreas muito próximas (Amorim et al. 2005, 2009; Moro et al. 2015b).

Em situação extrema encontra-se a vegetação que se desenvolve sobre os afloramentos rochosos onde se verifica reduzida disponibilidade de água, assim como escassez de solo devido à forte erosão hídrica (França et al. 1997). Os solos, ausentes ou muito reduzidos, resultantes do desgaste da rocha, criam micro-condições para a instalação de comunidades vegetais adaptadas a estas situações tão particulares. Os elencos florísticos destes ecossistemas são frequentemente distintos dos habitats vizinhos (Ribeiro et al. 2007) por se encontrarem sujeitos a fortes filtros ambientais, com adaptação ao estresse hídrico e às elevadas temperaturas (Moraes et al. 2009). Nestes ecossistemas de escassos recursos endógenos, a variação na composição da rocha determina a diversidade da sua morfologia que, por sua vez, afeta processos macro e micro-climatológicos, influenciando em conjunto a composição desta flora xerófila e rupícola (Porembski et al. 1994; Martinelli 2007) que pode ainda apresentar maior ou menor relação com a flora circundante (Burke 2002a,b). Se por um lado a umidade, a estabilidade da rocha e a disponibilidade de locais propícios para a germinação de sementes estão relacionados com maiores valores de diversidade e de densidade da vegetação nestes ecossistemas (Larson et al. 2000) por outro, o seu relativo isolamento e as condições muito específicas de substrato associadas a índices elevados de exposição solar e de amplitude térmica determinam maior número e quantidade de endêmicas (Porembski et al. 1996, 1998; Conceição et al. 2007a; Gomes \& Alves 2009; Pessoa \&
Alves 2014). Estas condições podem favorecer processos de especiação, e são determinantes na seleção das espécies que se desenvolvem nestes ambientes (Kluge \& Brulfert 2000; Conceição et al. 2007a; Porembski 2007). Por outro lado, estes ecossistemas funcionam como barreiras contra a invasão e o estabelecimento de plantas da vegetação adjacente, devido à reduzida retenção de água e às poucas alternativas para fixação de raízes e sementes (Larson et al. 2000; Burke 2002a,b). Nestes casos, para garantirem a sua propagação no espaço, as plantas necessitam de mecanismos de dispersão de longa distância (Costa et al. 2015).

Estas especificidades por si só justificam as análises de vegetação nestes ecossistemas. Por outro lado, a necessidade de conservação destes locais reforça a necessidade de se empreenderem esforços na sua caracterização detalhada para que assim se definam políticas de conservação bem adaptadas às realidades locais (Martinelli 2007; Scarano 2007; Gomes \& Sobral-Leite 2013). Neste estudo pretendeu-se efetuar a caracterização da flora e das comunidades vegetais que se desenvolvem em ambientes rupícolas (lajedos e inselbergues) no bioma Caatinga, associadas a afloramentos rochosos isolados e de baixa altitude e a situações edafo-xerófilas, circunscritos em três municípios da região norte cearense. A composição e a diferenciação florística e ecológica deste tipo de comunidades constituíram o principal objetivo deste estudo.

\section{Material e Métodos}

Localização das áreas de amostragem e caracterização geral das áreas de estudo

As estações estudadas selecionadas coincidem com grandes manchas de afloramentos rochosos presentes na região noroeste do Ceará. Selecionaram-se cinco áreas de estudo em diferentes fases de desenvolvimento (Fig. 1):

[A1] Serrote dos Picos (04 $01^{\circ} 34^{\prime} ' S$ e 4005'18'W, 190 m de altitude) em Santa Quitéria, CE;

[A2] Serrote dos Picos (04\%07'34"S e $40^{\circ} 08^{\prime} 01^{\prime \prime} \mathrm{W}, 180 \mathrm{~m}$ de altitude) em Santa Quitéria, CE;

[A3] Sítio Galinha de Angola (04\%09'35'S e 4009'33' W, 188 m de altitude) em Santa Quitéria, CE;

[A4] Unidade de Conservação Refúgio da Vida Silvestre Pedra da Andorinha (04 $03^{\circ}$ ' $35^{\prime}$ 'S e $40^{\circ} 00^{\prime}$ 39'W, 225 m de altitude) em Taperuaba, Sobral, CE;

[A5] Lagoa do Peixe (0356'39"S e $40^{\circ} 23^{\prime} 35^{\prime} \mathrm{W}, 111 \mathrm{~m}$ de altitude) em Groaíras, CE. 


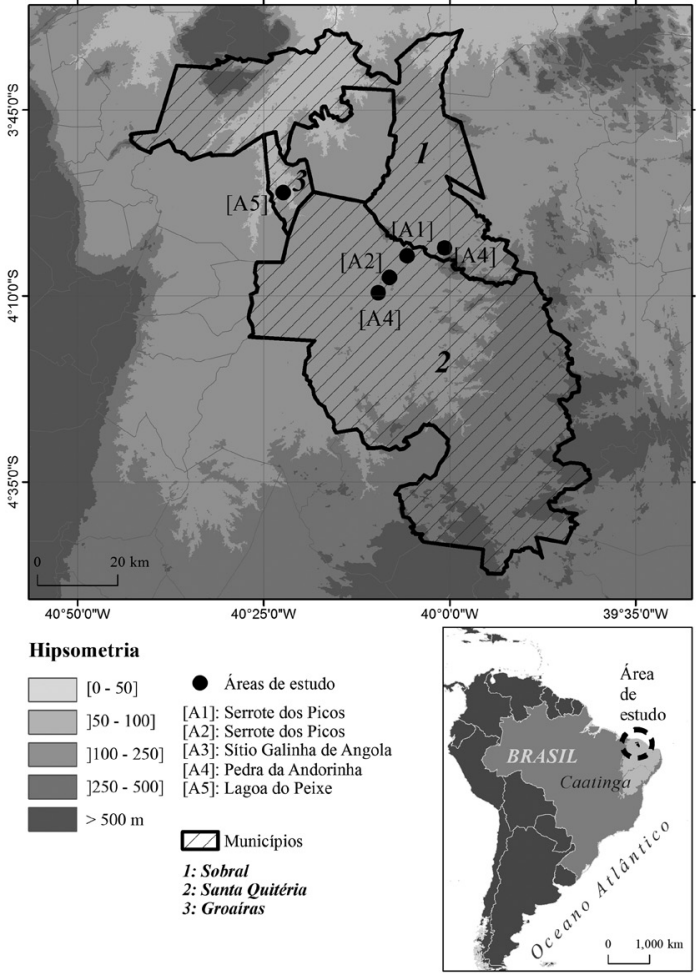

Figura 1 - Localização das áreas de estudo.

Figure 1 - Location of the study areas.

As áreas de estudo inserem-se na bacia hidrográfica do Rio Acaraú $\left(02^{\circ} 47^{\prime}\right.$ a $04^{\circ} 58^{\prime} \mathrm{S}$ e $39^{\circ} 40^{\prime}$ a $40^{\circ} 51^{\prime} \mathrm{W}$ ), que integra, entre outras, as sub-bacias de Aracatiaçu, Groaíras, Macacos, Madeiras e Jucurutu, com escoamentos em regimes intermitentes. O substrato geológico é constituído, fundamentalmente, por rochas do embasamento cristalino, representadas por gnaisses, migmatitos, granitos e xistos (Destro et al. 1994; Neves 1998). Segundo Moro et al. (2015a), o Ceará apresenta essencialmente dois grandes domínios geológicos, um sedimentar e outro dominado por litologias cristalinas que, ao longo dos tempos, foram modelados gerando distintos tipos de relevo. Na geomorfologia da região estudada de domínio cristalino destacam-se as superfícies aplanadas da Depressão Sertaneja, os relevos residuais associados (inselbergues), o maciço residual granítico da Serra da Meruoca, e a planície aluvial do Rio Acaraú (Peulvast \& Bétard 2015; Moro et al. 2015a; Rodrigues \& Lima 2015). Esta é uma região de contrastes, as altitudes variam na generalidade do território entre $90 \mathrm{~m}$ e $700 \mathrm{~m}$, podendo superar os $1.000 \mathrm{~m}$ na Serra da Meruoca (Peulvast \& Bétard 2015).
Os solos mais representativos da região estudada são os neossolos litólicos, solos rasos e geralmente pedregosos que ocorrem nas áreas mais acidentadas com afloramento rochosos e os luvissolos, solos rasos a pouco profundos e que apresentam frequentemente revestimento pedregoso na superfície (Cunha et al. 2010; Marques et al. 2014).

Segundo a classificação de Köppen, o clima predominante no Ceará é do tipo BSh de zona seca e que corresponde ao semiárido de baixa latitude e altitude, típico do Nordeste do Brasil, ocorrendo em regiões com precipitações médias anuais inferiores a $800 \mathrm{~mm}$ (Alvares et al. 2013). Nos três municípios a que pertencem as áreas estudadas este clima apresenta uma estação seca entre julho e janeiro, e uma estação chuvosa irregular entre fevereiro e junho, caracterizada por chuvas torrenciais (Sobrinho 2009; Alexandre et al. 2010). As temperaturas médias variam entre $19^{\circ} \mathrm{C}$ e $21{ }^{\circ} \mathrm{C}$ no inverno e entre $29^{\circ} \mathrm{C}$ e $36^{\circ} \mathrm{C}$ no verão, enquanto as precipitações anuais variam de 600 a $1.100 \mathrm{~mm}$ (Freise 1938; Werner \& Gerstengarbe 2003; Robertson et al. 2004; Sun et al. 2007; Santos et al. 2009).

De acordo com o esquema biogeográfico da América Latina e do Caribe proposto por Morrone (2001), o território estudado pertence à Região Neotropical, à Sub-região Chaquenha e à Província da Caatinga. A heterogeneidade evidenciada nas condições ambientais e as atividades antrópicas constituem os principais fatores que influenciam a distribuição espacial, estrutural e vegetacional no Ceará (Albuquerque et al. 2012; Moro et al. 2015a; Pereira et al. 2014; Silva et al. 2014).

A vegetação típica da Depressão Sertaneja é a Caatinga do cristalino, caduca na estação seca para evitar o estresse hídrico, espinhosa e adaptada ao clima semiárido com poucas espécies perenifólias (Moro et al. 2015a). Tendo por base o porte da vegetação, Figueiredo (1997) propõe três unidades fitoecológicas: a Floresta Caducifólia Espinhosa (Caatinga Arbórea), a Caatinga Arbustiva Densa e a Caatinga Arbustiva Aberta. $\mathrm{Na}$ área de estudo verifica-se o predomínio da Floresta Caducifólia Espinhosa em Groaíras e da Caatinga Arbustiva Aberta em Sobral e Santa Quitéria, surgindo pontualmente as matas úmidas e as matas ciliares sempre que existam condições ambientais específicas. Os ambientes rochosos encontram-se nos lajedos, terrenos planos que passam despercebidos na paisagem e cuja vegetação rupícola cresce sobre solos pouco 
desenvolvidos (neossolos litólicos) (Moro et al. 2015b).

Nas áreas de estudo aplicou-se o método de Braun-Blanquet ou clássico sigmatista (Ariza 2010; Braun-Blanquet 1979; Géhu \& Rivas-Martínez 1981) para a identificação de comunidades vegetais em habitats homogêneos, quer a nível fisionômico quer em condições ambientais. $\mathrm{Na}$ análise fitossociológica, o processo compreendeu duas etapas fundamentais (Ariza 2010; Rodal et al. 2013). A etapa analítica integra o delineamento amostral, a coleta de material vegetal e a identificação das espécies. O elenco florístico foi analisado de acordo com um conjunto de indicadores, entre os quais os tipos fisionómicos de Raunkiaer (1934). A etapa sintética culmina com a comparação dos dados entre unidades amostrais para classificação. Os diferentes procedimentos são seguidamente descritos com maior detalhe.

\section{Etapa analítica}

As coletas de material vegetal foram realizadas nos meses de março de 2014 e 2015, nos períodos chuvosos. As excursões contemplaram os afloramentos rochosos de baixa altitude (lajedos) e circunscritos nos municípios de Sobral, Groaíras e Santa Quitéria, num total de cinco áreas de
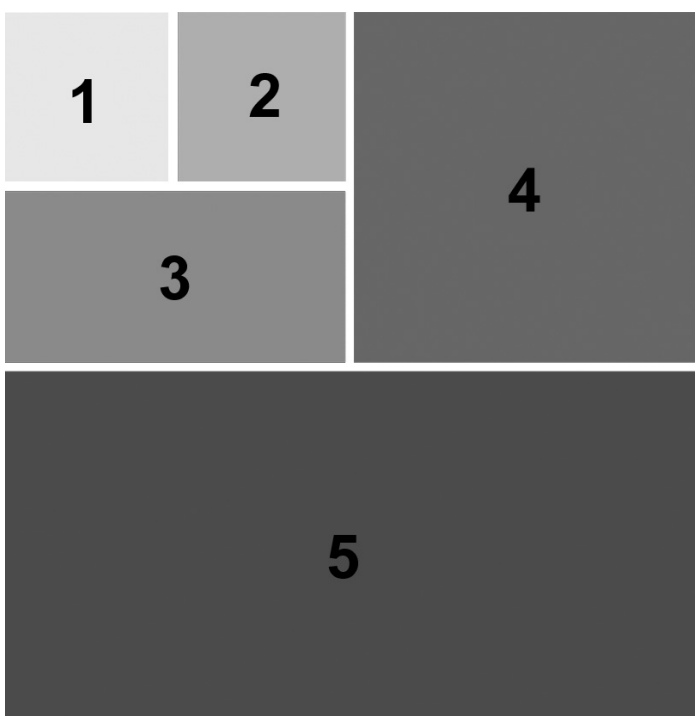

Figura 2 - Representação gráfica do cálculo da área mínima qualitativa de 1 a $16 \mathrm{~m}^{2}: 1=1 \times 1 \mathrm{~m}^{2} ; 2=2 \times 1$ $\mathrm{m}^{2} ; 3=2 \times 2 \mathrm{~m}^{2} ; 4=4 \times 2 \mathrm{~m}^{2} ; 5=4 \times 4 \mathrm{~m}^{2}$.

Figure 2 - Graphical representation of the calculation of qualitative minimum area from 1 to $16 \mathrm{~m}^{2}: 1=1 \times 1 \mathrm{~m}^{2} ; 2=2 \times$ $1 \mathrm{~m}^{2} ; 3=2 \times 2 \mathrm{~m}^{2} ; 4=4 \times 2 \mathrm{~m}^{2} ; 5=4 \times 4 \mathrm{~m}^{2}$. estudo. Foram incluídas no elenco florístico as espécies encontradas nas fissuras, fendas e ilhas de vegetação sobre afloramentos rochosos com elevada proporção de rocha exposta $(>80 \%)$, e excluíram-se as áreas ao redor dos lajedos, com Caatinga típica do cristalino, por não apresentarem o ambiente rochoso a estudar, devido a elevada percentagem de espécies não rupícolas.

Para garantir que a homogeneidade ecológica e florística de cada unidade amostral, e desta forma que as unidades amostrais (inventários) sejam claramente distinguíveis (Rodrigues 1988), definimos áreas homogéneas tendo por base as características edafoclimáticas do habitat, assim como a distribuição espacial das plantas, a variabilidade na dominância de grupos de plantas, e a ausência de mosaicos de zonas densas e esparsas e nos estratos de distribuição uniforme. Nas áreas previamente selecionadas, para além de identificados os diferentes tipos de comunidades pela composição florística (Ariza 2010), foi ainda avaliado o seu estado de conservação, de modo a evitar zonas degradadas e assim privilegiar áreas com reduzida influência antrópica.

Após a verificação destas condições efetuaram-se os inventários fitossociológicos, numa extensão suficiente para reunirem as espécies vegetais representativas de cada comunidade em estudo (Braun-Blanquet 1979). Assim, foi necessário determinar, para cada comunidade vegetal, uma área mínima correspondendo à parcela de menor superfície que congrega as espécies da comunidade vegetal em análise. Para a definição da área mínima recorreu-se ao aumento progressivo da área de amostragem, duplicada em progressão geométrica, a partir de uma área-base de $1 \times 1 \mathrm{~m}\left(1 \mathrm{~m}^{2}\right)$, até não se identificarem espécies novas a integrar no elenco florístico (Fig. 2).

Nos inventários fitossociológicos $(n=21)$, foram identificadas todas as espécies presentes na área mínima e, a cada uma atribuiu-se um índice de quantidade (Tab. 1), que resulta da combinação da abundância (frequência) e da dominância (ou cobertura) (Braun-Blanquet 1979).

O material botânico coletado foi identificado com auxílio da bibliografia especializada (Lorenzi 2008; Queiroz 2009; Prata et al. 2013), comparação morfológica com o material existente no Herbário Prof. Francisco José de Abreu Matos (HUVA) da Universidade Estadual Vale do Acaraú (UVA) em Sobral, Ceará, e consultas a especialistas. A sinonímia e a grafia dos nomes científicos foram efetuadas com base no BFG (2015). 
Tabela 1 - Índice de quantidade (abundânciadominância) (segundo o método de quantificação proposto por Braun-Blanquet 1979).

Table 1 - Abundance-dominance index (Braun-Blanquet 1979).

\begin{tabular}{cc}
\hline Índice & Significado \\
\hline+ & Indivíduos raros ou muito raros \\
1 & Indivíduos cobrindo menos de 5\% \\
2 & Indivíduos cobrindo de 5 a 25\% \\
3 & Indivíduos cobrindo de 25 a 50\% \\
4 & Indivíduos cobrindo de 50 a 75\% \\
5 & Indivíduos cobrindo mais de $75 \%$ \\
\hline
\end{tabular}

Indicadores de caracterização da flora

Para cada espécie da listagem florística identificou-se o nome comum, a forma de vida de Raunkier, a origem, o domínio fitogeográfico e o tipo de substrato.

A identificação das formas de vida de Raunkiaer (1934) para cada espécie foi feita a partir de diversos estudos (Araújo et al. 2008; Araújo et al. 2011; França et al. 2005; IBGE 2012). O sistema de Raunkier baseia-se no grau de proteção das gemas vegetativas das plantas vasculares na época desfavorável (Braun-Blanquet 1979; Martins \& Batalha 2011), classificandoas em cinco categorias: fanerófito, caméfito, hemicriptófito, terófito e geófito. Quanto à origem das espécies, aplicou-se a terminologia de Moro et al. (2012), onde usamos as categorias de "nativas", "nativas e endêmicas", e "exóticas-invasoras". Em relação à distribuição geográfica, as espécies foram agrupadas como ocorrendo nos seguintes domínios fitogeográficos: Caatinga; Caatinga e Cerrado; Caatinga e Mata Atlântica; e espécies de ampla distribuição (Amazônia, Caatinga, Cerrado, Mata Atlântica, Pampa, Pantanal). Estes dois últimos indicadores (origem e distribuição fitogeográfica) foram extraídos do BFG (2015) tal como o substrato que representa o tipo de suporte onde as plantas fixam as raízes (terrícola e/ou rupícola).

A distribuição dos indicadores mais relevantes de caracterização da flora foi analisada através de violin-plots através dos quais é possível observar o formato da distribuição de frequência dos indicadores (Hinze \& Nelson 1998). Os violin-plots foram construídos através da package “ggplot2” (Wickham 2016) para R versão 3.1.3 (R Development Core Team 2015).

\section{Etapa sintética}

A análise classificativa foi efetuada com recurso ao método TWINSPAN (two-way indicator species analysis), desenvolvido por Hill (1979). Na aplicação do algoritmo TWINSPAN recorreuse ao programa JUICE 7.0.33 (Tichý 2002). Este é um método adequado para os dados coletados e sintetizados de acordo com a metodologia descrita anteriormente. Para os grupos obtidos foi calculada a fidelidade (para identificação de espécies diagnóstico) através do coeficiente phi (Sokal \& Rohlf 1995; Chytrý et al. 2002) cuja significância estatística foi obtida através do teste exato de Fisher (Chytrý et al. 2002; Tichý $\&$ Chytrý 2006), com recurso ao programa JUICE (Tichý 2002). O conceito de espécies diagnóstico está relacionado com o conceito de fidelidade. Este avalia a concentração da ocorrência ou abundância de espécies dentro de um grupo ou comunidade tipo em relação ao restante conjunto de dados.

Posteriormente, os inventários fitossociológicos foram agrupados em tabelas, tendo em conta o tipo de habitat, reunindo-se igualmente um conjunto de dados auxiliares sobre o local amostrado: número de inventário, altitude média $(\mathrm{m})$, área mínima $\left(\mathrm{m}^{2}\right)$ e grau de cobertura (\%). No quadro fitossociológico, o nome da espécie foi abreviado a fim de evitar o uso de trinômios (e.g., Anadenanthera colubrina var. cebil (Griseb) Altschul para Anadenanthera cebil).

\section{Resultados}

Análise da flora existente

nos afloramentos rochosos

Foram registradas 88 espécies de plantas vasculares nas áreas de estudo, distribuídas em 59 gêneros e 30 famílias. Destacam-se as famílias Fabaceae (20 spp.), Poaceae (dez spp.), Euphorbiaceae (sete spp.) e Convolvulaceae (seis spp.) (Tab. 2).

A Figura 3 mostra a distribuição das duas famílias mais representativas, assim como da riqueza específica e das espécies endêmicas. A riqueza específica média é de 13 espécies. O valor mínimo neste indicador situou-se nas sete espécies (em três dos inventários realizados), enquanto o valor máximo atingiu as 23 espécies (embora este seja considerado um outlier na distribuição tal como pode ser observado na Fig. 3). Foram também identificadas 19 espécies endêmicas do Brasil 
Tabela 2 - Caracterização das espécies identificadas nas cinco áreas de estudo localizadas nos municípios de Santa Quitéria, Sobral e Groaíras.

NC: nome comum. FV: formas de vida de Raunkiaer - Fa - fanerófito; Ca- caméfito; He - hemicriptófito; Te - terófito. OR: origem - Nat - nativa; Nat-end - nativa e endémica; Exo-inv- exótica invasora. DF: domínios fitogeográficos - Caa - Caatinga; Caa/Ce - Caatinga e Cerrado; Caa/Ma - Caatinga e Mata Atlântica; Ad - ampla distribuição (Amazônia, Caatinga, Cerrado, Mata Atlântica, Pampa, Pantanal). SU: substrato - Te - terrícola; Ru - rupícola. AE: áreas de estudo - A1 - Serrote dos Picos (0401'34'S e 4005'18'W); A2 - Serrote dos Picos (0407'34'S e 4008'01'W); A3 - Sítio Galinha de Angola (0409'35"S e 4009'33"W); A4 - Unidade de Conservação Refúgio da Vida Silvestre

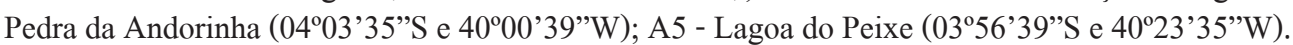

Table 2 - Characterization of the identified species in the five study areas located in the municipalities of Santa Quiteria, Sobral and Groaíras.

$\mathrm{CN}$ : common name. FV: life forms of Raunkiær - Fa - phanerophyte; Ca - chamaephyte; He - hemicryptophyte; Te - therophyte. OR: origin - Nat - native; Nat-end - native and endemic; Exo-inv - invasive alien. DG: phytogeographic domains - Caa - Caatinga; Caa/Ce - Caatinga and Cerrado; Caa/Ma - Caatinga and Mata Atlântica; Ad - wide distribution (Amazônia, Caatinga, Cerrado, Mata Atlântica,

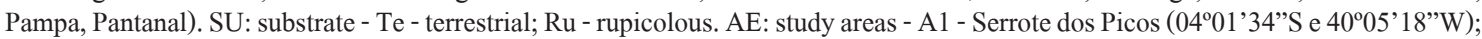

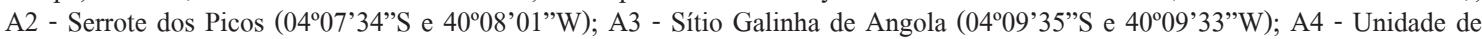

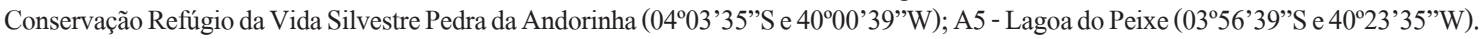

\begin{tabular}{|c|c|c|c|c|c|c|c|c|c|c|}
\hline \multirow{2}{*}{ Família/espécie } & \multirow{2}{*}{ NC } & \multirow{2}{*}{ FV } & \multirow{2}{*}{ OR } & \multirow{2}{*}{ DF } & \multirow{2}{*}{ SU } & \multicolumn{5}{|c|}{$\mathbf{A E}$} \\
\hline & & & & & & A1 & $\mathbf{A 2}$ & A3 & A4 & A5 \\
\hline \multicolumn{11}{|l|}{ Amaranthaceae } \\
\hline Alternanthera brasiliana (L.) Kuntze & cabeça-branca & $\mathrm{Te}$ & Nat & Ad & Ter & $\mathrm{X}$ & $\mathrm{X}$ & & & $\mathrm{X}$ \\
\hline Alternanthera tenella Colla & apaga-fogo & $\mathrm{Te}$ & Nat & Ad & Ter & & & & $\mathrm{X}$ & $\mathrm{X}$ \\
\hline $\begin{array}{l}\text { Froelichia humboldtiana (Roem. \& } \\
\text { Schult.) Seub. }\end{array}$ & ervaço & $\mathrm{Te}$ & Nat & $\mathrm{Caa} / \mathrm{Ce}$ & Ter & $\mathrm{X}$ & & $\mathrm{X}$ & $\mathrm{X}$ & \\
\hline \multicolumn{11}{|l|}{ Asclepiadaceae } \\
\hline Ditassa hastata Decne. & - & $\mathrm{Ca}$ & Nat-end & Ad & $\mathrm{Ru}$ & & & & $\mathrm{X}$ & \\
\hline \multicolumn{11}{|l|}{ Asteraceaea } \\
\hline Bidens bipinnata L. & - & $\mathrm{Te}$ & Exo-inv & Ad & Ter & & & & & $\mathrm{X}$ \\
\hline \multicolumn{11}{|l|}{ Bignoniaceae } \\
\hline $\begin{array}{l}\text { Amphilophium crucigerum (L.) L.G. } \\
\text { Lohmann }\end{array}$ & pente-de-macaco & $\mathrm{Fa}$ & Nat & Ad & Ter & & & & $\mathrm{X}$ & \\
\hline \multicolumn{11}{|l|}{ Boraginaceaea } \\
\hline Cordia oncocalyx Allemão & pau-branco & $\mathrm{Fa}$ & Nat-end & $\mathrm{Caa}$ & Ter & & & $\mathrm{X}$ & & $\mathrm{X}$ \\
\hline \multicolumn{11}{|l|}{ Bromeliaceae } \\
\hline $\begin{array}{l}\text { Encholirium spectabile Mart. ex } \\
\text { Schult. \& Schult.f. }\end{array}$ & $\begin{array}{l}\text { macambira-de- } \\
\text { flecha }\end{array}$ & $\mathrm{Ca}$ & Nat-end & Ad & $\mathrm{Ru}$ & & & $\mathrm{X}$ & $\mathrm{X}$ & \\
\hline \multicolumn{11}{|l|}{ Cactaceae } \\
\hline Cereus jamacaru DC. & mandacaru & $\mathrm{Fa}$ & Nat-end & $\mathrm{Caa} / \mathrm{Ce}$ & $\mathrm{Ter} / \mathrm{Ru}$ & & & & $\mathrm{X}$ & \\
\hline $\begin{array}{l}\text { Pilosocereus chrysostele (Vaupel) } \\
\text { Byles \& G.D.Rowley }\end{array}$ & facheiro & $\mathrm{Fa}$ & Nat-end & $\mathrm{Caa}$ & $\mathrm{Ru}$ & & & & $\mathrm{X}$ & \\
\hline $\begin{array}{l}\text { Pilosocereus gounellei (FA.C.Weber) } \\
\text { Byles \& Rowley }\end{array}$ & xique-xique & $\mathrm{Fa}$ & Nat-end & $\mathrm{Caa} / \mathrm{Ce}$ & $\mathrm{Ter} / \mathrm{Ru}$ & $\mathrm{X}$ & $\mathrm{X}$ & $\mathrm{X}$ & $\mathrm{X}$ & $\mathrm{X}$ \\
\hline \multicolumn{11}{|l|}{ Capparaceae } \\
\hline Crateva tapia $\mathrm{L}$. & trapiá & $\mathrm{Fa}$ & Nat & Ad & Ter & & $\mathrm{X}$ & & $\mathrm{X}$ & \\
\hline \multicolumn{11}{|l|}{ Cleomaceae } \\
\hline Physostemon guianense (Aubl.) Malme & - & $\mathrm{Te}$ & Nat & Ad & Ter & & & $\mathrm{X}$ & & \\
\hline \multicolumn{11}{|l|}{ Combretaceae } \\
\hline Combretum leprosum Mart. & mofumbo & $\mathrm{Fa}$ & Nat & Ad & Ter & $\mathrm{X}$ & $\mathrm{X}$ & $\mathrm{X}$ & $\mathrm{X}$ & \\
\hline \multicolumn{11}{|l|}{ Commelinaceae } \\
\hline Commelina difusa Burm.f. & trapoeraba-azul & $\mathrm{Te}$ & Nat & $\mathrm{Ad}$ & Ter & & & & $\mathrm{X}$ & \\
\hline
\end{tabular}




\begin{tabular}{|c|c|c|c|c|c|c|c|c|c|c|}
\hline \multirow{2}{*}{ Família/espécie } & \multirow{2}{*}{ NC } & \multirow{2}{*}{ FV } & \multirow{2}{*}{ OR } & \multirow{2}{*}{ DF } & \multirow{2}{*}{$\mathbf{S U}$} & \multicolumn{5}{|c|}{$\mathbf{A E}$} \\
\hline & & & & & & A1 & A2 & A3 & A4 & A5 \\
\hline \multicolumn{11}{|l|}{ Convolvulaceae } \\
\hline Evolvulus alsinoides L. & - & $\mathrm{He}$ & Nat & Ad & Ter & & & $\mathrm{X}$ & & \\
\hline Evolvulus filipes Mart. & - & $\mathrm{Te}$ & Nat & Ad & Ter & & & $\mathrm{X}$ & & $\mathrm{X}$ \\
\hline Ipomoea decipiens Dammer & - & $\mathrm{He}$ & Nat & $\mathrm{Caa} / \mathrm{Ma}$ & Ter & & & & & $\mathrm{X}$ \\
\hline Ipomoea piurensis O’Donell & - & $\mathrm{He}$ & Nat & Ad & Ter & & & & & $\mathrm{X}$ \\
\hline $\begin{array}{l}\text { Jacquemontia evolvuloides (Moric.) } \\
\text { Meisn. }\end{array}$ & - & $\mathrm{Ca}$ & Nat & Ad & Ter & & $\mathrm{X}$ & $\mathrm{X}$ & & $\mathrm{X}$ \\
\hline $\begin{array}{l}\text { Jacquemontia gracilima( Choisy) } \\
\text { Hallier f. }\end{array}$ & - & $\mathrm{Ca}$ & Nat-end & Ad & Ter & & & $\mathrm{X}$ & $\mathrm{X}$ & \\
\hline \multicolumn{11}{|l|}{ Cyperaceae } \\
\hline Cyperus eragrostis Lam. & capim-navalha & $\mathrm{He}$ & Nat & Ad & Ter & & $\mathrm{X}$ & & $\mathrm{X}$ & $\mathrm{X}$ \\
\hline Cyperus uncinulatus Schrad. ex Nees & barba-de- bode & $\mathrm{He}$ & Nat & Ad & Ter & & & & & $\mathrm{X}$ \\
\hline Pycreus lanceolatus (Poir.) C.B.Clarke & junca & $\mathrm{He}$ & Nat & Ad & Ter & $\mathrm{X}$ & & & & \\
\hline \multicolumn{11}{|l|}{ Euphorbiaceae } \\
\hline Croton campestris A. St.-Hil. & $\begin{array}{l}\text { velame-do- } \\
\text { campo }\end{array}$ & $\mathrm{Fa}$ & Nat & Ad & Ter & $\mathrm{X}$ & & & & \\
\hline Croton heliotropiifolius Kunth & velame & $\mathrm{Fa}$ & Nat & Ad & Ter & $\mathrm{X}$ & & & & \\
\hline Croton hirtus L'Hér. & - & $\mathrm{Te}$ & Nat & Ad & Ter & & $\mathrm{X}$ & $\mathrm{X}$ & $\mathrm{X}$ & $\mathrm{X}$ \\
\hline Croton sonderianus Müll. Arg. & marmeleiro-preto & $\mathrm{Fa}$ & Nat-end & Caa & Ter & $\mathrm{X}$ & $\mathrm{X}$ & $\mathrm{X}$ & & $\mathrm{X}$ \\
\hline Dalechampia scandens L. & urtiga-mamão & $\mathrm{He}$ & Nat & Ad & Ter & $\mathrm{X}$ & & & $\mathrm{X}$ & \\
\hline $\begin{array}{l}\text { Euphorbia bahiensis (Klotzsch \& } \\
\text { Garcke) Boiss. }\end{array}$ & - & $\mathrm{He}$ & Nat & Ad & Ter & $\mathrm{X}$ & $\mathrm{X}$ & $\mathrm{X}$ & & $\mathrm{X}$ \\
\hline Jatropha mollissima (Pohl) Baill. & pinhão-bravo & $\mathrm{Fa}$ & Nat & Ad & Ter & & $\mathrm{X}$ & & $\mathrm{X}$ & $\mathrm{X}$ \\
\hline \multicolumn{11}{|l|}{ Fabaceae } \\
\hline $\begin{array}{l}\text { Amburana cearensis (Allemão ) } \\
\text { A.C.Smith }\end{array}$ & $\begin{array}{l}\text { imburana-de- } \\
\text { cheiro }\end{array}$ & $\mathrm{Fa}$ & Nat & Ad & Ter & & & & & $\mathrm{X}$ \\
\hline $\begin{array}{l}\text { Anadenanthera colubrina var. cebil } \\
\text { (Griseb) Altschul }\end{array}$ & angico & $\mathrm{Fa}$ & Nat & Ad & Ter & & & $\mathrm{X}$ & & \\
\hline Arachis repens Handro & grama-amendoim & $\mathrm{Te}$ & Nat-end & Ad & Ter & $\mathrm{X}$ & & & & \\
\hline Centrosema brasilianum (L.) Benth. & feijão-de-rolinha & $\mathrm{He}$ & Nat & Ad & Ter & & & & & $\mathrm{X}$ \\
\hline $\begin{array}{l}\text { Centrosema pascuorum Mart. ex } \\
\text { Benth. }\end{array}$ & centrosema & $\mathrm{Te}$ & Nat & Ad & Ter & & $\mathrm{X}$ & $\mathrm{X}$ & $\mathrm{X}$ & $\mathrm{X}$ \\
\hline $\begin{array}{l}\text { Chamaecrista amiciella (H.S.Irwin \& } \\
\text { Barneby) H.S.Irwin \& Barneby }\end{array}$ & - & $\mathrm{Ca}$ & Nat-end & $\mathrm{Caa} / \mathrm{Ce}$ & Ter & & $\mathrm{X}$ & & & \\
\hline $\begin{array}{l}\text { Chamaecrista calycioides (DC. ex } \\
\text { Collad.) Greene }\end{array}$ & - & $\mathrm{He}$ & Nat & Ad & Ter & & & & $\mathrm{X}$ & \\
\hline $\begin{array}{l}\text { Chamaecrista linearis (H.S.Irwin \& } \\
\text { Barneby) Afr.Fern. \& E.P.Nunes }\end{array}$ & - & $\mathrm{Ca}$ & Nat-end & Ad & Ter & & $\mathrm{X}$ & & $\mathrm{X}$ & \\
\hline Chamaecrista nictitans (L.) Moench & mata-pasto & $\mathrm{Ca}$ & Nat & Ad & Ter & $\mathrm{X}$ & & & & \\
\hline Macroptilium lathyroides (L.) Urb. & feijão-de-rola & $\mathrm{Te}$ & Nat & Ad & Ter & $\mathrm{X}$ & & & & \\
\hline Mimosa caesalpiniifolia Benth. & sabiá & $\mathrm{Fa}$ & Nat-end & Ad & Ter & & $\mathrm{X}$ & & & \\
\hline Mimosa pudica L. & malícia & $\mathrm{Ca}$ & Nat & Ad & Ter & $\mathrm{X}$ & $\mathrm{X}$ & $\mathrm{X}$ & $\mathrm{X}$ & $\mathrm{X}$ \\
\hline Mimosa sensitiva $\mathrm{L}$. & unha-de-gato & $\mathrm{Ca}$ & Nat & Ad & Ter & $\mathrm{X}$ & & & & \\
\hline Mimosa tenuiflora (Willd.) Poir. & jurema-preta & $\mathrm{Fa}$ & Nat & $\mathrm{Caa} / \mathrm{Ce}$ & Ter & & & & $\mathrm{X}$ & \\
\hline $\begin{array}{l}\text { Poincianella bracteosa (Tul.) } \\
\text { L.P.Queiroz }\end{array}$ & catingueira & $\mathrm{Fa}$ & Nat-end & $\mathrm{Caa} / \mathrm{Ce}$ & Ter & & & $\mathrm{X}$ & & \\
\hline $\begin{array}{l}\text { Poincianella pyramidalis (Tul.) } \\
\text { L.P.Queiroz }\end{array}$ & catingueira & $\mathrm{Fa}$ & Nat & Ad & Ter & & & & $X$ & \\
\hline
\end{tabular}

Rodriguésia 69(2): 281-299. 2018 


\begin{tabular}{|c|c|c|c|c|c|c|c|c|c|c|}
\hline \multirow{2}{*}{ Família/espécie } & \multirow{2}{*}{ NC } & \multirow{2}{*}{ FV } & \multirow{2}{*}{ OR } & \multirow{2}{*}{ DF } & \multirow{2}{*}{ SU } & \multicolumn{5}{|c|}{$\mathbf{A E}$} \\
\hline & & & & & & A1 & A2 & A3 & A4 & A5 \\
\hline $\begin{array}{l}\text { Senna obtusifolia (L.) H.S.Irwin \& } \\
\text { Barneby }\end{array}$ & besouro & $\mathrm{Ca}$ & Nat & Ad & Ter & & & & & $\mathrm{X}$ \\
\hline Stylosanthes guianensis (Aubl.) Sw. & vassourinha & $\mathrm{Ca}$ & Nat & Ad & Ter & $\mathrm{X}$ & & & & \\
\hline Stylosanthes humilis Kunth & erva-da-ovelha & $\mathrm{Te}$ & Nat & Ad & Ter & & $\mathrm{X}$ & $\mathrm{X}$ & $\mathrm{X}$ & $\mathrm{X}$ \\
\hline Zornia brasiliensis Vogel & urinária & $\mathrm{Te}$ & Nat & Ad & Ter & & & & $\mathrm{X}$ & \\
\hline \multicolumn{11}{|l|}{ Hydroleaceae } \\
\hline Hydrolea spinosa L. & $\begin{array}{l}\text { carqueja-do- } \\
\text { pântano }\end{array}$ & $\mathrm{Ca}$ & Nat & Ad & Ter & & & $\mathrm{X}$ & & \\
\hline \multicolumn{11}{|l|}{ Lamiaceae } \\
\hline $\begin{array}{l}\text { Marsypianthes chamaedrys (Vahl) } \\
\text { Kuntze }\end{array}$ & hortelã-do-campo & $\mathrm{Te}$ & Nat & Ad & Ter & & & & & $\mathrm{X}$ \\
\hline Mesosphaerum suaveolens (L.) Kuntze & bamburral & $\mathrm{Te}$ & Nat & Ad & Ter & $\mathrm{X}$ & $\mathrm{X}$ & $\mathrm{X}$ & & \\
\hline Vitex polygama Cham. & azeitona do campo & $\mathrm{Fa}$ & Nat-end & Ad & Ter & & & $\mathrm{X}$ & & \\
\hline \multicolumn{11}{|l|}{ Malvaceae } \\
\hline Sida cordifolia $\mathrm{L}$. & malva-branca & $\mathrm{Te}$ & Nat & Ad & Ter & & & $\mathrm{X}$ & & \\
\hline Sida galheirensis Ulbr. & $\begin{array}{l}\text { malva-branca- } \\
\text { cabeluda }\end{array}$ & $\mathrm{Ca}$ & Nat-end & Ad & Ter & & & $\mathrm{X}$ & & \\
\hline Sida spinosa $\mathrm{L}$. & $\begin{array}{l}\text { guanxuma-de- } \\
\text { espinho }\end{array}$ & $\mathrm{Fa}$ & Nat & Ad & Ter & & $\mathrm{X}$ & & $\mathrm{X}$ & $\mathrm{X}$ \\
\hline Waltheria operculata Rose & - & $\mathrm{Ca}$ & Nat & Ad & Ter & & $\mathrm{X}$ & $\mathrm{X}$ & $\mathrm{X}$ & $\mathrm{X}$ \\
\hline \multicolumn{11}{|l|}{ Olacaceae } \\
\hline Ximenia americana $\mathrm{L}$. & $\begin{array}{l}\text { ameixa-de- } \\
\text { espinho }\end{array}$ & $\mathrm{Fa}$ & Nat & Ad & Ter & & $\mathrm{X}$ & & $\mathrm{X}$ & \\
\hline \multicolumn{11}{|l|}{ Oxalidaceae } \\
\hline Oxalis cratensis Oliv. ex Hook. & erva-imbú & $\mathrm{Te}$ & Nat & Ad & Ter & & & & & $\mathrm{X}$ \\
\hline Oxalis divaricata Mart. ex Zucc. & mato-azedo & $\mathrm{Ca}$ & Nat-end & Ad & Ter & $\mathrm{X}$ & & $\mathrm{X}$ & & \\
\hline \multicolumn{11}{|l|}{ Passifloraceae } \\
\hline Passiflora cincinata Mast. & $\begin{array}{l}\text { maracujá-do- } \\
\text { mato }\end{array}$ & $\mathrm{Fa}$ & Nat & Ad & Ter & & & $\mathrm{X}$ & & \\
\hline Passiflora foetida $\mathrm{L}$. & $\begin{array}{l}\text { maracujá-de- } \\
\text { estalo }\end{array}$ & $\mathrm{Ca}$ & Nat & Ad & Ter & $\mathrm{X}$ & $\mathrm{X}$ & $\mathrm{X}$ & & \\
\hline \multicolumn{11}{|l|}{ Phyllanthaceae } \\
\hline Phyllanthus tenellus Roxb. & quebra-pedra & $\mathrm{Ca}$ & Nat & Ad & Ter & & & & & $\mathrm{X}$ \\
\hline \multicolumn{11}{|l|}{ Phytolaccaceae } \\
\hline Microtea glochidiata Moq. & - & $\mathrm{Te}$ & Nat-end & Ad & Ter & & $\mathrm{X}$ & & & $\mathrm{X}$ \\
\hline Microtea paniculata Moq. & - & $\mathrm{Te}$ & Nat & $\mathrm{Ad}$ & Ter & & & $\mathrm{X}$ & $\mathrm{X}$ & \\
\hline \multicolumn{11}{|l|}{ Poaceae } \\
\hline Aristida adscensionis L. & $\begin{array}{l}\text { capim-panasco- } \\
\text { verdadeiro }\end{array}$ & $\mathrm{Te}$ & Exo-inv & Ad & Ter & & $\mathrm{X}$ & & $\mathrm{X}$ & \\
\hline Aristida setifolia Kunth & - & $\mathrm{Te}$ & Nat & Ad & Ter & $\mathrm{X}$ & & $\mathrm{X}$ & $\mathrm{X}$ & \\
\hline Chloris gayana Kunth & capim-rhodes & $\mathrm{He}$ & Nat & Ad & Ter & & $\mathrm{X}$ & & & \\
\hline Digitaria horizontalis Willd. & capim-de-roça & $\mathrm{Te}$ & Exo-inv & Ad & Ter & $\mathrm{X}$ & & & & \\
\hline Eragrostis lehmaniana Nees & - & $\mathrm{He}$ & Exo-inv & Caa & Ter & & & $\mathrm{X}$ & $\mathrm{X}$ & \\
\hline Homolepis isocalycia (G.Mey.) Chase & - & $\mathrm{He}$ & Nat & Ad & Ter & & & & & $\mathrm{X}$ \\
\hline Melinis repens (Willd.) Zizka & capim-favorito & $\mathrm{Te}$ & Exo-inv & Ad & Ter & & & & $\mathrm{X}$ & \\
\hline $\begin{array}{l}\text { Paspalum convexum Humb. \& Bonpl. } \\
\text { ex Flüggé }\end{array}$ & - & $\mathrm{Te}$ & Nat & Ad & Ter & & & & $\mathrm{X}$ & $\mathrm{X}$ \\
\hline
\end{tabular}




\begin{tabular}{|c|c|c|c|c|c|c|c|c|c|c|}
\hline \multirow{2}{*}{ Família/espécie } & \multirow{2}{*}{$\mathrm{NC}$} & \multirow{2}{*}{ FV } & \multirow{2}{*}{ OR } & \multirow{2}{*}{ DF } & \multirow{2}{*}{ SU } & \multicolumn{5}{|c|}{$\mathbf{A E}$} \\
\hline & & & & & & A1 & A2 & A3 & A4 & A5 \\
\hline Paspalum virgatum $\mathrm{L}$. & capim-navalha & $\mathrm{He}$ & Nat & Ad & Ter & & $\mathrm{X}$ & & $\mathrm{X}$ & $\mathrm{X}$ \\
\hline $\begin{array}{l}\text { Urochloa mollis (Sw.) Morrone \& } \\
\text { Zuloaga }\end{array}$ & - & $\mathrm{Te}$ & Exo-inv & Ad & Ter & & & & & $\mathrm{X}$ \\
\hline \multicolumn{11}{|l|}{ Polygalaceae } \\
\hline $\begin{array}{l}\text { Asemeia violacea (Aubl.) J.F.B.Pastore } \\
\& \text { J.R.Abbott }\end{array}$ & roxinha & $\mathrm{Te}$ & Nat & Ad & Ter & $\mathrm{X}$ & & & & $\mathrm{X}$ \\
\hline \multicolumn{11}{|l|}{ Portulacaceae } \\
\hline Portulaca elatior Mart. & - & $\mathrm{Te}$ & Nat & Ad & $\mathrm{Ru}$ & & $\mathrm{X}$ & $\mathrm{X}$ & & $\mathrm{X}$ \\
\hline Portulaca oleracea L. & $\begin{array}{l}\text { beldroega-da- } \\
\text { horta }\end{array}$ & $\mathrm{Te}$ & Nat & $\mathrm{Ad}$ & Ter & & $\mathrm{X}$ & $\mathrm{X}$ & & \\
\hline \multicolumn{11}{|l|}{ Rubiaceae } \\
\hline $\begin{array}{l}\text { Diodella apiculata (Willd. ex Roem. \& } \\
\text { Schult.) Delprete }\end{array}$ & - & $\mathrm{Ca}$ & Nat & Ad & $\mathrm{Ru}$ & & $\mathrm{X}$ & $\mathrm{X}$ & & \\
\hline Diodella teres (Walter) Small & $\begin{array}{l}\text { quebra-tijela-de- } \\
\text { folha-estreita }\end{array}$ & $\mathrm{Te}$ & Nat & Ad & Ter & & & & & $\mathrm{X}$ \\
\hline \multicolumn{11}{|l|}{ Turneraceae } \\
\hline Piriqueta duarteana (Cambess.) Urb. & - & $\mathrm{Ca}$ & Nat-end & Ad & Ter & $\mathrm{X}$ & & & & \\
\hline Turnera pumilea $\mathrm{L}$. & - & $\mathrm{Ca}$ & Nat & Ad & Ter & & $\mathrm{X}$ & $\mathrm{X}$ & & $\mathrm{X}$ \\
\hline Turnera subulata Sm. & chanana & $\mathrm{Ca}$ & Nat & Ad & Ter & & $\mathrm{X}$ & & & \\
\hline \multicolumn{11}{|l|}{ Verbenaceae } \\
\hline $\begin{array}{l}\text { Stachytarpheta cayennensis (Rich.) } \\
\text { Vahl }\end{array}$ & gervão-de-folha & $\mathrm{Te}$ & Nat & Ad & Ter & $\mathrm{X}$ & & & & \\
\hline Stachytarpheta microphylla Walp. & chá-do-brasil & $\mathrm{Te}$ & Nat-end & $\mathrm{Caa}$ & Ter & $\mathrm{X}$ & & & & \\
\hline \multicolumn{11}{|l|}{ Violaceae } \\
\hline $\begin{array}{l}\text { Pombalia communis (A.St.-Hil.) Paula- } \\
\text { Souza }\end{array}$ & - & $\mathrm{Ca}$ & Nat & Ad & Ter & & & & $\mathrm{X}$ & $\mathrm{X}$ \\
\hline
\end{tabular}

(21,6\% do total de espécies identificadas): Arachis repens, Cereus jamacaru, Chamaecrista amiciella, Chamaecrista linearis, Cordia oncocalyx, Croton sonderianus, Ditassa hastata, Encholirium spectabile, Jacquemontia gracilis, Microtea glochidiata, Mimosa caesalpiniifolia, Oxalis divaricata, Pilosocereus chrysostele, Pilocereus gounellei, Piriqueta duarteana, Poincianella bracteosa, Sida galheirensis, Stachytarpheta microphylla e Vitex polygama. O número médio de espécies endêmicas do Brasil em cada inventário é 3 , tendo sido encontrados 3 inventários que podem ser considerados outliers, com 5, 6 e 7 espécies endêmicas respetivamente (Fig. 3).

As formas de vida de Raunkiaer dominantes foram os terófitos (35,2\%), seguidos pelos caméfitos $(25,0 \%)$, fanerófitos $(23,9 \%)$ e hemicriptófitos $(15,9 \%)$ (Tab. 3), não tendo sido amostrado nenhum geófito (Fig. 4).

Esta evidência pode ainda ser observada na Tabela 4, com o espectro para cada uma das cinco áreas de estudo, e na Figura 5, onde estão distribuídas as formas de vida de Raunkiaer em cada inventário apresentado nas Tabelas A2 e A3 (disponibilizadas no link $<$ https://doi.org/10.6084/ m9.figshare.5915233.v2>).

Em relação à ocorrência das espécies nos domínios fitogeográficos, verificou-se que $5,7 \%$ das espécies (Cordia oncocalyx, Croton sonderianus, Eragrostis lehmaniana, Pilosocereus chrysostele e Stachytarpheta microphylla) encontram-se restritas à Caatinga, 6,8\% (Cereus jamacaru, Chamaecrista amiciella, Froelichia humboldtiana, Mimosa tenuiflora, Pilosocereus gounellei e Poincianella bracteosa) ocorrem na Caatinga e Cerrado, 1,1\% (Ipomoea decipiens ) na Caatinga e Mata Atlântica e 86,4\% são de ampla distribuição geográfica (Amazônia, Caatinga, Cerrado, Mata Atlântica, Pampa e Pantanal) (Tab. 3). Em relação à origem, $71,6 \%$ são nativas, $21,6 \%$ nativas e endêmicas do Brasil e 6,8\% são exóticas invasoras (Aristida adscensionis, Bidens bipinnata, Digitaria 
Tabela 3 - Formas de vida, origem, domínios fitogeográficos e tipo de substrato das áreas de estudo (municípios de Santa Quitéria, Sobral e Groaíras).

FV: formas de vida de Raunkiaer - Fa - fanerófito, Ca - caméfito, He - hemicriptófito, Te - terófito. OR: origem - Nat - nativa, Nat-end - nativa e endémica, Exo-inv - exótica invasora. DF: domínios fitogeográficos - Caa - Caatinga; Caa/Ce - Caatinga e Cerrado; Caa/Ma - Caatinga e Mata Atlântica; Ad - ampla distribuição (Amazônia, Caatinga, Cerrado, Mata Atlântica, Pampa, Pantanal). SU: substrato - Ter - terrícola, Ru - rupícola. ne: número de espécies, $\%$ : percentagens.

Table 3 - Life forms, origin, phytogeographic domains and substrate type and substrate type of study areas (municipalities of Santa Quitéria, Sobral and Groaíras).

FV: life forms of Raunkiær - Fa - phanerophyte, $\mathrm{Ca}$ - chamaephyte, He - hemicryptophyte, Te - therophyte. OR: origin - Nat - native, Nat-end - native and endemic, Exo-inv - invasive alien. DF: phytogeographic domains - Caa - Caatinga, Caa/Ce - Caatinga and Cerrado, Caa/Ma - Caatinga and Mata Atlântica, Ad - wide distribution (Amazônia, Caatinga, Cerrado, Mata Atlântica, Pampa, Pantanal). SU: substrate - Ter - terrestrial; Ru - rupicolous. ne: number of specie, \%: percentages.

\begin{tabular}{|c|c|c|c|c|c|c|c|c|c|c|c|}
\hline FV & ne & $\%$ & OR & ne & $\%$ & DF & ne & $\%$ & SU & ne & $\%$ \\
\hline $\mathrm{Fa}$ & 21 & 23,9 & Nat & 63 & 71,6 & $\mathrm{Caa}$ & 5 & 5,7 & Ter & 81 & 92,0 \\
\hline $\mathrm{Ca}$ & 22 & 25,0 & Nat-end & 19 & 21,6 & $\mathrm{Caa} / \mathrm{Ce}$ & 6 & 6,8 & $\mathrm{Ru}$ & 5 & 5,7 \\
\hline $\mathrm{He}$ & 14 & 15,9 & Exo-inv & 6 & 6,8 & $\mathrm{Caa} / \mathrm{Ma}$ & 1 & 1,1 & $\mathrm{Ter} / \mathrm{Ru}$ & 2 & 2,3 \\
\hline $\mathrm{Te}$ & 31 & 35,2 & & & & $\mathrm{Ad}$ & 76 & 86,4 & & & \\
\hline Total & 88 & 100 & Total & 88 & 100 & Total & 88 & 100 & Total & 88 & 100 \\
\hline
\end{tabular}

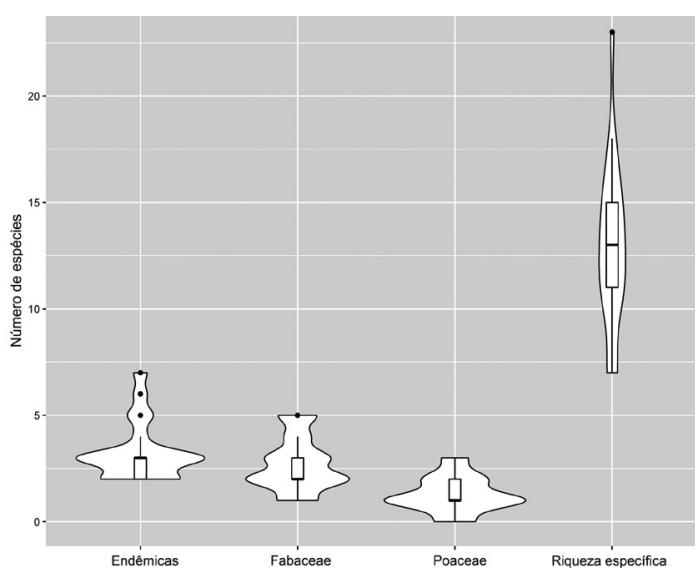

Figura 3 - Violin-plot com a distribuição, por inventário, das espécies endêmicas, das leguminosas, das gramíneas e da riqueza específica.

Figure 3 - Violin-plot of the distribution, by relevés, of endemic species, Fabaceae, Poaceae and specific richness.

horizontalis, Eragrostis lehmaniana, Melinis repens e Urochloa mollis) (Tab. 3).

Quanto ao tipo de substrato, 92,0\% são reportadas na Flora do Brasil como terrícolas, 5,7\% como rupícolas e 2,3\% como rupícolas/terrícolas (Tab. 3).

Na Tabela 5 é apresentada a riqueza específica e o número de endemismos presentes nas estações estudadas. Verificou-se que a estação 5 na Lagoa do Peixe em Groaíras, embora com reduzida margem,

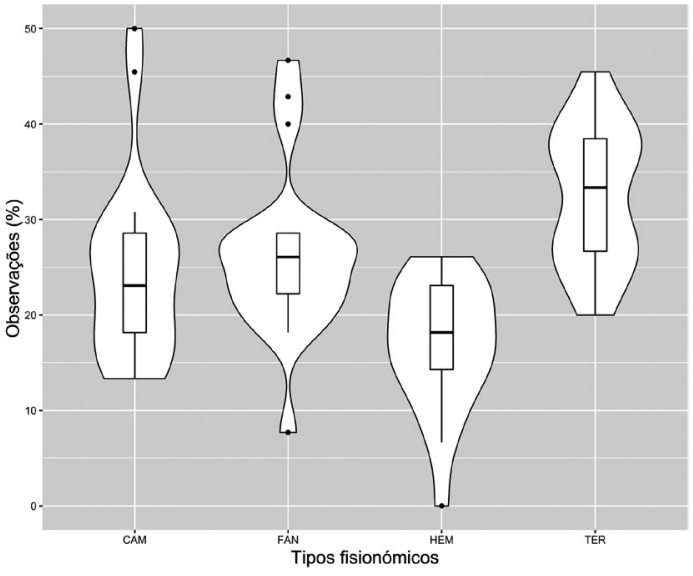

Figura 4 - Violin-plot com a distribuição dos tipos fisionómicos de Raunkiaer (1934). CAM: caméfitos; FAN: fanerófitos; HEM: hemicriptófitos; TER: terófitos. Figure 4 -Violin-plot of the distribution of life forms sensu Raunkiaer (1934). CAM: chamaephytes; FAN: phanerophytes; HEM: hemicryptophytes; TER: therophytes.

apresentou o valor mais elevado (40,9\%) enquanto na riqueza em endemismos foi a estação 3 , com 9 espécies, no Sítio Galinha de Angola em Santa Quitéria.

\section{Análise da vegetação}

Foram realizados 21 inventários fitossociológicos de acordo com o método de Braun-Blanquet ou clássico sigmatista (Tab. $6 \mathrm{e}$ Tabs. A2 e A3, disponibilizadas no link < https://doi. 
Tabela 4 - Espectro das formas de vida de Raunkiaer para cada área de estudo (\%) - Te = terófitos; Ca = caméfitos; $\mathrm{He}=$ hemicriptófitos; $\mathrm{Fa}=$ fanerófitos. $\mathrm{A} 1=$ Serrote dos Picos $\left(4^{\circ} 1^{\prime} 34^{\prime}\right.$ 'S, $\left.40^{\circ} 5^{\prime} 18^{\prime \prime} \mathrm{W}\right) ; \mathrm{A} 2=$ Serrote dos Picos (4'7'34' 'S, 40 8' 1' 'W); A3 = Sítio Galinha de Angola (49'35'’S, 409'33' 'W); A4 = Unidade de Conservação Refúgio

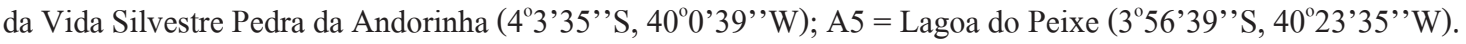
Table 4 - Raunkiaer life-form spectra for each study area (\%). Te = therophytes; $\mathrm{Ca}=$ chamaephytes; $\mathrm{H}=$ hemicryptophytes; $\mathrm{Fa}=$ phanerophytes. A1 = Serrote dos Picos (4'1'34',S, 40 5'18' 'W); A2 = Serrote dos Picos (4'7'34''S, 40 8' '1' 'W); A3 = Sítio Galinha de Angola (4 9'35'”S, 40 9'33'”W); A4 = Unidade de Conservação Refúgio da Vida Silvestre Pedra da Andorinha (4 3'35' 'S, 400'39' 'W); A5 = Lagoa do Peixe (35' $39^{\prime \prime}$ S, $\left.40^{\circ} 23^{\prime} 35^{\prime \prime} \mathrm{W}\right)$.

\begin{tabular}{cccccc}
\hline Áreas de estudo & Te & Ca & He & Fa & \\
\hline A1 & 40,00 & 28,00 & 12,00 & 20,00 & 100 \\
A2 & 30,00 & 30,00 & 13,33 & 26,67 & 100 \\
A3 & 35,30 & 32,35 & 8,82 & 23,53 & 100 \\
A4 & 34,29 & 20,00 & 14,29 & 31,42 & 100 \\
A5 & 41,67 & 19,44 & 22,22 & 16,67 & 100 \\
\hline TOTAL & 35.23 & 25.00 & 15.91 & 23.86 & 100 \\
\hline
\end{tabular}

org/10.6084/m9.figshare.5915233.v2>). Nas áreas estudadas, determinaram-se as áreas mínimas de 8 e/ou $16 \mathrm{~m}^{2}$ para as áreas ocupadas pela vegetação rupícola de fissuras e fendas das rochas e pelas ilhas de vegetação em áreas mais alargadas e com maior espessura de solo respetivamente.

Os inventários foram agrupados com base na semelhança florística e reunidos em tabelas (Tabs. A2 e A3, disponibilizadas no link < https:// doi.org/10.6084/m9.figshare.5915233.v2>). No cabeçalho inscreveram-se as informações referentes a cada um dos inventários, nas linhas horizontais todas as espécies presentes nas áreas mínimas e nas colunas verticais, os respetivos índices de quantidade, por ordem decrescente de presenças.
A classificação TWINSPAN separou os inventários em 5 grupos (Fig. 6), aos quais reconhecemos a correspondência de duas comunidades: A - Comunidade de Pilosocereus gounellei e Encholirium spectabile de fendas e fissuras rochosas nos municípios de Santa Quitéria, Sobral e Groaíras; e B - Comunidade de Crateva tapia e Combretum leprosum das ilhas de vegetação nos afloramentos rochosos nos municípios de Santa Quitéria e Sobral.

Na Tabela A1 (disponibilizada no link $<$ https://doi.org/10.6084/m9.figshare.5915233. v2>) apresenta-se a separação dos grupos individualizados no dendrograma da Figura 6. A comunidade A foi ainda subdivididida no grupo 1 e 3 (Fig. 6 e Tab. A1, (disponibilizada no link

Tabela 5 - Riqueza específica e espécies endémicas nas áreas estudadas (municípios de Santa Quitéria, Sobral e Groaíras). AE: Áreas de estudo. RE: riqueza específica. EN: espécies endêmicas.

Table 5 - Specific richness and endemic species in the study areas (municipalities of Santa Quitéria, Sobral and Groaíras). AE: Study areas. RE: specific richness. EN: endemic species.

\begin{tabular}{|c|c|c|}
\hline $\mathbf{A E}$ & $\mathbf{R E}$ & EN \\
\hline A1 & 26 & $\begin{array}{l}\text { Arachis repens, Croton sonderianus, Oxalis divaricata, Piriqueta duarteana, Pilosocereus gounellei, } \\
\text { Stachytarpheta microphylla }\end{array}$ \\
\hline A2 & 30 & $\begin{array}{l}\text { Chamaecrista amiciella, Chamaecrista linearis, Croton sonderianus, Microtea glochidiata, Mimosa } \\
\text { caesalpiniifolia, Pilosocereus gounellei }\end{array}$ \\
\hline $\mathbf{A 3}$ & 34 & $\begin{array}{l}\text { Cordia oncocalyx, Croton sonderianus, Encholirium spectabile, Jacquemontia gracilis, Oxalis divaricata, } \\
\text { Pilosocereus gounellei, Poincianella bracteosa, Sida galheirensis, Vitex polygama }\end{array}$ \\
\hline A4 & 35 & $\begin{array}{l}\text { Cereus jamacaru, Chamaecrista linearis, Ditassa hastata, Encholirium spectabile, Jacquemontia gracilis, } \\
\text { Pilosocereus chrysostele, Pilosocereus gounellei }\end{array}$ \\
\hline A5 & 36 & Cordia oncocalyx, Croton sonderianus, Microtea glochidiata, Pilosocereus gounellei \\
\hline
\end{tabular}


Tabela 6 - Distribuição do número de inventários realizados em cada área de estudo e respetivas áreas mínimas. AE: Áreas de estudo. AM: área mínima. NIR: número de inventários realizados em cada área de estudo. NI: número do inventário de acordo com as Tabelas A2 e A3 do Anexo.

Table 6 - Distribution of the number of relevés carried out in each study area and respective minimum area.

AE: Study areas. AM: minimum area. NIR: number of relevés carried out in each study area. NI: number of the relevés according with the Tables A2 and A3 included in the Annex.

\begin{tabular}{llccc}
\hline & AE & AM & NIR & NI \\
\hline$[\mathrm{A} 1]$ & Serrote dos Picos & 8 e $16 \mathrm{~m}^{2}$ & 7 & $1-4 ; 14-16$ \\
{$[\mathrm{~A} 2]$} & Serrote dos Picos & 8 e $16 \mathrm{~m}^{2}$ & 1 & 5 \\
{$[\mathrm{~A} 3]$} & Sítio Galinha de Angola & $8 \mathrm{e} 16 \mathrm{~m}^{2}$ & 4 & $6-8 ; 17$ \\
{$[\mathrm{~A} 4]$} & Unidade de Conservação Refúgio da Vida Silvestre Pedra da Andorinha & $16 \mathrm{~m}^{2}$ & 5 & $9 ; 18-21$ \\
{$[\mathrm{~A} 5]$} & Lagoa do Peixe & $16 \mathrm{~m}^{2}$ & 4 & $10-13$ \\
\hline
\end{tabular}

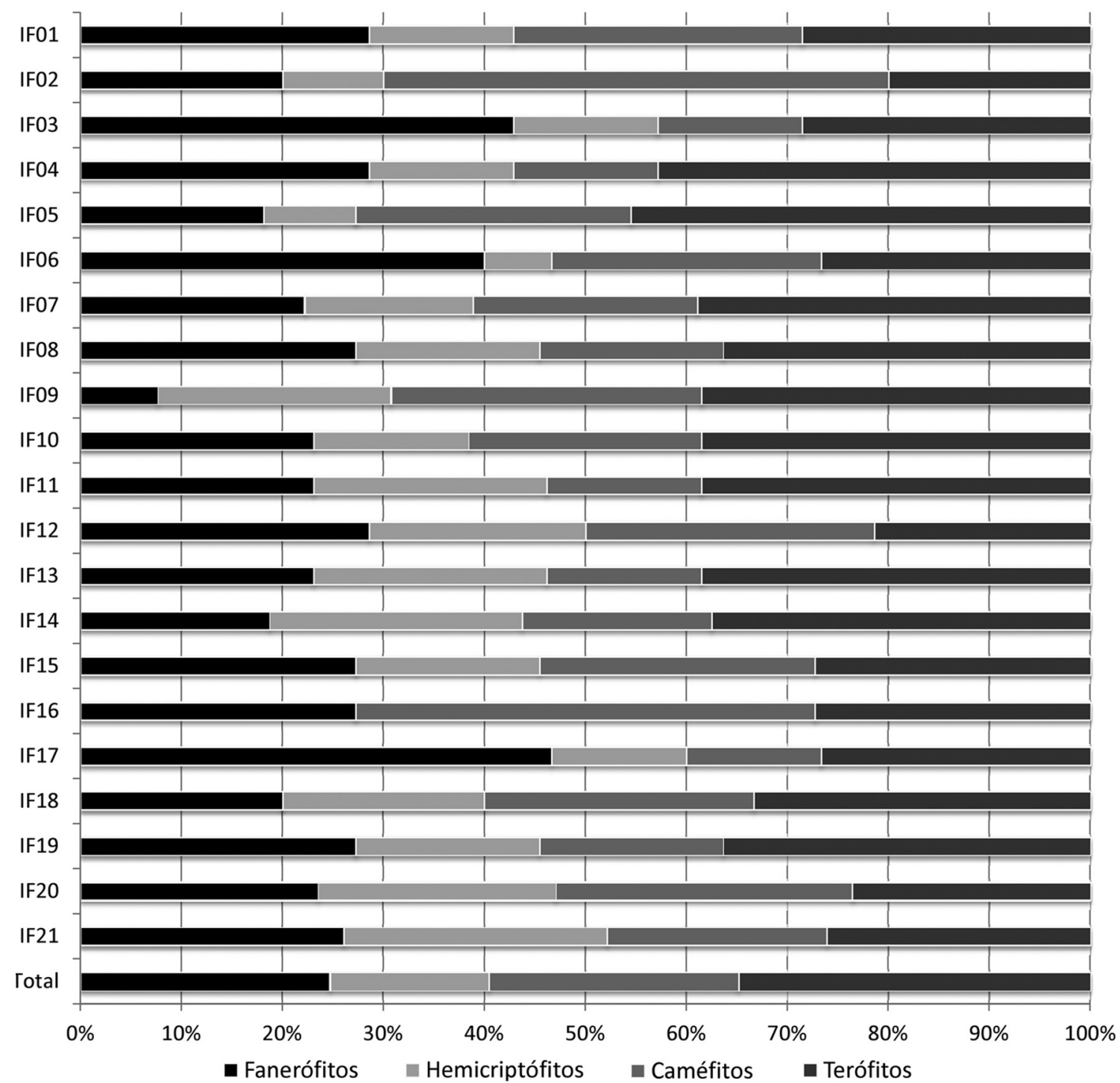

Figura 5 - Distribuição, por inventário, dos tipos fisionómicos de Raunkiaer (1934).

Figure 5 - Distribution, by relevés, of life forms sensu Raunkiaer (1934). 


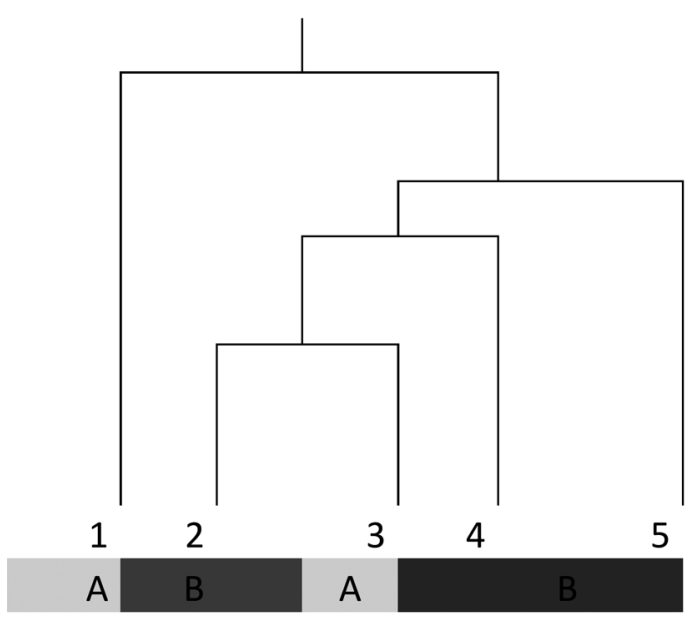

Figura 6 - Dendrograma da classificação TWINSPAN. A - Comunidade de Pilosocereus gounellei e Encholirium spectabile; B - Comunidade de Crateva tapia e Combretum leprosum.

Figure 6 - Dendrogram of TWINSPAN classification. A Community Pilosocereus gounellei and Encholirium spectabile community; B - Crateva tapia and Combretum leprosum community.

$<$ https://doi.org/10.6084/m9.figshare.5915233. v2>). Estas subdivisões refletem a presença de espécies de comunidades de contato, inclusive, com a comunidade B. Assim sendo, o grupo 3 ficou separado do grupo 1 da comunidade A pelo fato de incorporar algumas espécies da comunidade B, na sua composição florística. Esta situação poderse-á justificar pela localização do afloramento rochoso (A5 - Lagoa do Peixe, Groaíras) numa área dominada pela Floresta Caducifólia Espinhosa (umbrófila) e pelo microclima mais fresco e úmido.

A tabela sinóptica (Tab.A1, (disponibilizada no link $<$ https://doi.org/10.6084/m9.figshare.5915233. v2 $>$ ) mostra que a fidelidade obtida para a espécie Crateva tapia tem elevada frequência e fidelidade significativa na comunidade $\mathrm{B}$, sendo uma das espécies que melhor diferencia a comunidade $\mathrm{A}$ da comunidade B.

\section{Comunidade de Pilosocereus gounellei} e Encholirium spectabile

Trata-se de uma comunidade que cresce nas fendas e fissuras dos afloramentos rochosos de baixa altitude, cujos habitats são caracterizados pelo reduzido teor de umidade e pouco solo acumulado. São formações altas e espinhosas, com variação em altura de 0,30 a $2 \mathrm{~m}$, xerófilas, muito resistentes à seca, circunscritas aos afloramentos rochosos, dominadas pelo Pilosocereus gounellei (xique-xique) e onde se destaca o Encholirium spectabile (macambira-de-flecha) na área de estudo 3 (Sítio Galinha de Angola, Santa Quitéria) (Tabs. A1 e A2, disponibilizadas no link < https:// doi.org/10.6084/m9.figshare.5915233.v2>). Além do Pilosocereus gounellei e Encholirium spectabile, foram identificadas outras espécies rupícolas tais como Physostemon guianense, Portulaca elatior e Turnera pumilea (Tab. A2, (disponibilizada no link $<$ https://doi.org/10.6084/ m9.figshare.5915233.v2>).

As espécies companheiras constituem um elenco florístico bastante diversificado de árvores e arbustos de pequenas dimensões (frutescens) que crescem, nas ilhas de vegetação (depressões entre rochas) onde existe substrato suficiente para enraizarem, como o Combretum leprosum (mofumbo), Cordia oncocalyx (paubranco), Croton heliotropiifolius (velame), Croton hirtus, Croton sonderianus (marmeleiro-preto), Jatropha molissima (pinhão-bravo) e Poincianella bracteosa (catingueira), acompanhados por vários subarbustos, herbáceas e lianas (Tab. A2, disponibilizada no link < https://doi.org/10.6084/ m9.figshare.5915233.v2>).

\section{Comunidade de Crateva tapia e Combretum leprosum}

Nas ilhas de vegetação, cujas áreas mínimas variaram de 10 a $16 \mathrm{~m}^{2}$ e com substrato suficiente para enraizarem pequenas árvores e arbustos, encontram-se manchas (patches) de comunidades pioneiras rústicas, capazes de sobreviver em condições muito adversas. No início da sucessão, estas ilhas foram colonizadas por espécies efêmeras das comunidades circunvizinhas e posteriormente, quando o substrato foi ficando mais profundo começaram a aparecer as pioneiras com maior porte, as arbustivas e as arbóreas, pouco exigentes em solos férteis (Figueiredo 2010). Fazem parte deste grupo as espécies identificadas nas áreas de estudo 2 (Serrote dos Picos), 3 (Sítio Galinha de Angola) e 4 (Unidade de Conservação Refúgio da Vida Silvestre Pedra da Andorinha): Anadenanthera colubrina var. cebil (angico), Crateva tapia (trapiá), Cereus jamacaru (mandacaru), Combretum leprosum (mofumbo), Cordia oncocalyx (pau-branco), Mimosa tenuiflora (jurema-preta), Poincianella bracteosa (catingueira), Ximenia americana (ameixa-deespinho) (Tab. A3, disponibilizada no link $<$ https:// doi.org/10.6084/m9.figshare.5915233.v2>). 
$\mathrm{Na}$ análise da Tab. A3 (disponibilizada no link $<$ https://doi.org/10.6084/m9.figshare.5915233. v2 $>$ ), destaca-se uma comunidade dominada por Crateva tapia (trapiá) e Combretum leprosum (mofumbo), por serem duas espécies muito resistentes em situações desfavoráveis.

As espécies companheiras são pequenos arbustos, subarbustos e herbáceas que pertencem a várias famílias botânicas que se encontram na envolvência dos afloramentos rochosos (Tab. A3, disponibilizada no link <https://doi.org/10.6084/ m9.figshare.5915233.v2>).

\section{Discussão}

A pertinência da análise apresentada deve-se não só à elevada diversidade existente no bioma Caatinga (Moro et al. 2014), mas também devido à necessidade de se efetuarem análises focadas nos habitats associados aos afloramentos rochosos em geral, tal como anteriormente referido por vários autores (Meirelles et al. 1999; Caiafa \& Silva 2007; Gomes et al. 2011), em particular nas ilhas de vegetação aí existentes (Meirelles et al. 1999). Os afloramentos rochosos constituem uma barreira seletiva à ocupação e estabelecimento de espécies vegetais. Neles, os habitats rupícolas caracterizamse principalmente pela ausência e/ou escassez de solo, pelas características edáficas, por fatores micro-climatológicos limitantes potenciados por características geomorfológicas e pela natureza geológica das rochas (e.g., Abreu et al. 2012; Arruda et al. 2015; Carlucci et al. 2015).

Para a área de estudo foram identificadas 89 espécies pertencentes a 60 gêneros e 31 famílias botânicas. Fabaceae é a família que apresenta o maior número de espécies (20 spp.), a Poaceae com dez spp., Euphorbiaceae com sete spp. e Convolvulaceae com 6 spp. Estes resultados são coincidentes com vários estudos que enfatizam estas famílias dentre as mais representativas em vegetação rupestre na Caatinga (Araújo et al. 2005; Gomes \& Alves 2010; Gomes et al. 2011; Tölke et al. 2011; Sales-Rodrigues et al. 2014). No entanto, os resultados obtidos não evidenciam a presença de Orchidaceae, e apenas foi identificada uma espécie da família Bromeliaceae ao contrário de outros trabalhos que salientam a elevada abundância de espécies destas duas famílias (Porto et al. 2008; Gomes \& Alves 2010; Pessoa \& Alves 2014; Silva et al. 2015). Uma vez que o objetivo central era testar o método fitossociológico na identificação das comunidades rupícolas, as amostragens concentraram-se num período fenológico apenas, sendo que uma amostragem mais exaustiva ao longo do ano poderá aumentar o elenco florístico destas áreas.

$\mathrm{Na}$ análise global das formas de vida de Raunkier, assim como nos espectros das áreas de estudo quando analisadas isoladamente, os terófitos são dominantes, seguidos pelos fanerófitos, caméfitos e hemicriptófitos. Outros trabalhos evidenciam o domínio dos terófitos neste tipo de ecossistemas na Floresta Atlântica, na Pedra do Trevo (Catende) e no Lajedo do Uruçu (Maraial), no estado de Pernambuco no Nordeste do Brasil (Gomes \& Sobral-Leite 2013), ou de fanerófitos e terófitos na região semiárida do nordeste brasileiro, também no estado de Pernambuco (Gomes \& Alves 2010). Ao contrário dos resultados obtidos neste estudo, Conceição et al. (2007b) encontraram um predomínio de fanerófitos e hemicriptófitos em afloramentos rochosos quartzíticos na Chapada Diamantina, no estado da Bahia. De acordo com a meta-análise publicada recentemente por Moro et al. (2016) na Caatinga do cristalino e nos inselbergues da Caatinga os terófitos são dominantes, enquanto a Caatinga do sedimentar apresenta uma dominância dos fanerófitos.

Foram também encontradas 19 espécies endêmicas do Brasil. Apesar de alguns afloramentos rochosos estarem próximos de zonas urbanas - Sítio da Galinha da Angola em Santa Quitéria e Lagoa do Peixe em Groaíras - não se verificaram, nas comunidades rupícolas a presença de espécies nitrófilas.

Propõe-se duas comunidades fitossociológicas em lajedos da Caatinga na região estudada, a de "Pilosocereus gounellei e Encholirium spectabile" de fendas e fissuras rochosas (Tab. A2, disponibilizada no link < https://doi.org/10.6084/ m9.figshare.5915233.v2>) e a de Crateva tapia e Combretum leprosum das ilhas de vegetação entre os afloramentos rochosos (Tab. A3, disponibilizada no link <https://doi.org/10.6084/ m9.figshare.5915233.v2>).

Em relação à primeira comunidade, Taylor \& Zappi (2002) referem que na distribuição das espécies da família Cactaceae no Brasil, o Pilosocereus gounellei (xiquexique) é uma espécie exclusiva da Caatinga. Desenvolve-se nas áreas mais secas da região semiárida do Nordeste, em solos delgados e multiplica-se regularmente nos afloramentos rochosos, cobrindo extensas áreas da Caatinga principalmente nos estados do Ceará, Rio Grande do Norte e Bahia (Gomes 1977), sendo uma das espécies mais comuns no bioma (Moro et al. 
2014). De um modo geral, esta formação encontrase em condições edafoclimáticas caracterizadas por elevadas temperaturas, precipitações pluviométricas irregulares e baixa fertilidade natural do solo (Taylor \& Zappi 2002). Na época da seca, o xiquexique em particular, é utilizado na pecuária como uma alternativa forrageira na alimentação de ruminantes (Silva et al. 2005, 2011), em que a parte aérea da planta é cortada e queimada para eliminação dos espinhos, e posteriormente fornecida aos animais (Cavalcanti $\&$ Resende 2007). Em algumas regiões a queima do xique-xique é feita no local onde crescem e os animais consomem diretamente no campo. Esta prática tem causado sérios danos à vegetação de lagedo, porque as plantas queimadas acabam por morrer (Cavalcanti \& Resende 2007).

Encholirium spectabile também se encontra restrita aos afloramentos rochosos em regiões do semiárido do Nordeste brasileiro (Ramalho et al. 2004), nos estados do Piauí, Rio Grande do Norte, Ceará, Paraíba, Pernambuco, Sergipe e Bahia (Smith \& Downs 1974; Forzza et al. 2003, 2005; BFG 2015) em solos areno-pedregosos ou afloramentos rochosos (Forzza 2001).

Foi inventariada na área de estudo 3 (Sítio Galinha de Angola - Santa Quitéria) em agrupamentos monospecíficos cobrindo em 2015, menos de 5\% das áreas mínimas (inventários fitossociológicos 6, 7 e 8 da Tab. 2, disponibilizada no link <https://doi.org/10.6084/ $\mathrm{m} 9$.figshare.5915233.v2>). Este táxon que foi avaliado como de menos interesse por Forzza (2003), não é beneficiado por proteção legal no Brasil. O fogo, a mineração, o pastoreio e a coleta de espécimes desta bromeliacea não controlados, poderão contribuir para a degradação acelerada do seu habitat natural.

A segunda comunidade, dominada por Crateva tapia e Combretum leprosum foi inventariada em áreas mínimas que oscilaram de 8 a $16 \mathrm{~m}^{2}$ (Tab. A3 do Anexo), nas ilhas de vegetação com substrato suficiente para o enraizamento de árvores e arbustos provenientes das comunidades circunvizinhas pouco exigentes em solos férteis (Figueiredo 2010). O trapiá (Crateva tapia) é uma espécie nativa amplamente distribuída no Brasil (norte, nordeste, centro-oeste e sudeste), comum nos domínios da Amazônia, Caatinga, Cerrado, e Mata Atlântica (Cornejo \& Iltis 2008). Apresenta copa arredondada e densa, sendo recomendada para arborização e restauração de áreas degradadas por ação antrópica (Silva et al. 2007). A espécie neotropical Combretum leprosum (mofumbo) tem distribuição exclusiva na América do Sul e encontra-se na Bolívia, Paraguai (Loiola et al. 2009), nos estados do norte e nordeste brasileiro, Mato Grosso e Mato Grosso do Sul (Chaves et al. 2007). Está considerada como espécie pioneira, semidecídua, xerófita e heliófila, além de ser utilizada também na recuperação de áreas degradadas (Maia 2004).

Numa escala mais alargada onde estejam incluídos inselbergues, que são grandes rochas ou grupo de rochas monolíticas que emergem acima da planície (e.g., Gomes \& Alves 2009; Moro et al. 2015a; Sales-Rodrigues et al. 2014). Esta classificação deverá ser alterada pela inclusão de novas espécies adaptadas à altitude, à composição da rocha, à forma do relevo e à biogeografia, entre outros fatores, além de justificar a ausência, nos lajedos estudados, de espécies das famílias Orchidaceae e Bromeliaceae (apenas foi identificada uma espécie desta última) (França et al. 1997; Gomes \& Alves 2009). Por outro lado, o elenco florístico destes afloramentos rochosos foi condicionado pela época de coleta (março de 2014 e 2015), e constituiu-se como uma limitação deste estudo. As variadas perturbações antrópicas e pastoreio mais ou menos acentuadas poderão igualmente ter efeitos na distribuição espacial e na diversidade das espécies (Silva et al. 2014; Pereira et al. 2014). Estas observações evidenciam a necessidade de reforço na aquisição de conhecimento sobre estes ecossistemas para que assim seja possível suportar o desenvolvimento de estratégias de conservação adaptadas às realidades de cada local em função da maior ou menor especificidade (ou raridade) das comunidades vegetais (e animais que por elas são suportados), do regime de perturbações ecológicas ou ainda de mudanças expectáveis no clima (Leal et al. 2005; Albuquerque et al. 2012; Oliveira et al. 2012; Silva et al. 2014).

\section{Agradecimentos}

Os autores agradecem aos estudantes do Curso de Ciências Biológicas/UVA, Ana Carolina Brasileiro Melo, Dalyne Menezes Teles, Daniela Bastos Ramos, José Alves, Maria Tereza Albuquerque Alves; assim como ao Sr. Lourival Vieira da Silva da Fazenda da UVA, e à Dr. ${ }^{a}$ Maria Vilma Gomes Mendes da Unidade de Conservação Refúgio da Vida Silvestre Pedra da Andorinha, que colaboraram nas saídas de campo, e à Universidade Estadual Vale do Acaraú (UVA), 
o apoio logístico durante a estadia da primeira autora no Brasil. Os autores agradecem ainda os comentários e sugestões dos revisores e do editor. Este trabalho foi parcialmente financiado por Fundos FEDER através do Programa Operacional Factores de Competitividade - COMPETE e por Fundos Nacionais através da FCT - Fundação para a Ciência e a Tecnologia, no âmbito do Financiamento Estratégico UID/AGR/04129/2013 concedido ao Centro de Investigação em Agronomia, Alimentos, Ambiente e Paisagem do Instituto Superior de Agronomia (Universidade de Lisboa), e UID/ AGR/00115/2013 concedido ao Instituto de Ciências Agrárias e Ambientais Mediterrânicas.

\section{Referências}

Abreu MF, Pinto JRR, Maracahipes L, Gomes L, Oliveira EA, Marimon BS, Marimon Junior BH, Farias J \& Lenza E (2012) Influence of edaphic variables on the floristic composition and structure of the tree-shrub vegetation in typical and rocky outcrop cerrado areas in Serra Negra, Goiás State, Brazil. Brazilian Journal of Botany 35: 259-272.

Albuquerque UP, Araújo EL, El-Deir ACA, Lima ALA, Souto A, Bezerra BM, Ferraz EMN, Freire EMX, Sampaio EVSB, Las-Casas FMG, Moura GJB, Pereira GA, Melo JG, Ramos MA, Rodal MJN, Schiel N, Lyra-Neves RM, Alves RRN, AzevedoJúnior SM, Telino Júnior WR \& Severi W (2012) Caatinga revisited: ecology and conservation of an important seasonal dry forest. The Scientific World Journal 2012: 205182.

Alexandre DMB, Andrade EMD, Lopes FB, Palácio HAQ \& Ferreira ACDS (2010) The water quality investigation using GIS and multivariable analysis in a semiarid region reservoir. Revista Ciência Agronômica 41: 554-561.

Alvares CA, Stape JL, Sentelhas PC, Moraes Gonçalves JL \& Sparovek G (2013) Köppen's climate classification map for Brazil. Meteorologische Zeitschrift 22: 711-728.

Amorim IL, Sampaio EVSB \& Araujo EL (2009) Fenologia de espécies lenhosas da caatinga do Seridó, RN. Revista Árvore 33: 491-499.

Amorim IL, Sampaio EVSB \& Araújo EL (2005) Flora e estrutura da vegetação arbustivo-arbórea de uma área de caatinga do Seridó, RN, Brasil. Acta Botanica Brasilica 19: 615-623.

Apgaua DMG, Santos RM, Pereira DGS, Menino GCO, Pires GG, Fontes MAL \& Tng DYP (2014) Betadiversity in seasonally dry tropical forests (SDTF) in the Caatinga Biogeographic Domain, Brazil, and its implications for conservation. Biodiversity and Conservation 23: 217-232.

Araújo EL, Silva KA, Ferraz EMN, Sampaio EVSB \& Silva SI (2005) Diversidade de herbáceas em microhabitats rochoso, plano e ciliar em uma área de caatinga, Caruaru, PE, Brasil. Acta Botanica Brasilica 19: 285-294.

Araújo FS, Costa RC, Lima JR, Vasconcelos SF, Girão LC, Sobrinho MS, Bruno MMA, Souza SSG, Nunes EP, Figueiredo MA, Lima-Verde LW \& Loiola MIB (2011) Floristics and life-forms along a topographic gradient, central-western Ceará, Brazil. Rodriguésia 62: 341-366.

Araújo FS, Oliveira RF \& Lima-Verde LW (2008) Composição, espectro biológico e síndromes de dispersão da vegetação de um inselbergue no domínio da Caatinga, Ceará. Rodriguésia 59: 659-671.

Ariza FJA (2010) El método fitosociológico. In: Geobotánica Tema 13. Universidad de Murcia. España. Disponível em $<$ http://www.um.es/docencia/ geobotanica/ficheros/tema13.pdf $>$. Acesso em 6 maio 2016.

Arruda DM, Schaefer CE, Corrêa GR, Rodrigues PM, Duque-Brasil R, Ferreira-Júnior WG \& OliveiraFilho AT (2015) Landforms and soil attributes determine the vegetation structure in the Brazilian semiarid. Folia Geobotanica 50: 175-184.

BFG - The Brazil Flora Group (2015) Growing knowledge: an overview of seed plant diversity in Brazil. Rodriguésia 66: 1085-1113.

Braun-Blanquet J (1979) Fitosociologia. Bases para el estudio de las comunidades vegetales. Blume, Madrid. 820p.

Burke A (2002a) Island-matrix relationships in Nama Karoo inselberg landscapes Part I: do inselbergs provide a refuge for matrix species? Plant Ecology 160: 79-90.

Burke A (2002b) Island-matrix relationships in Nama Karoo inselberg landscapes Part II: are some inselbergs better sources than others? Plant Ecology 158: 41-48.

Caiafa AN \& Silva AFD (2007) Structural analysis of the vegetation on a highland granitic rock outcrop in Southeast Brazil. Brazilian Journal of Botany 30: 657-664.

Carlucci MB, Bastazini VA, Hofmann GS, Macedo JH, Iob G, Duarte LD, Hartz SM \& Müller SC (2015) Taxonomic and functional diversity of woody plant communities on opposing slopes of inselbergs in southern Brazil. Plant Ecology \& Diversity 8: 187-197.

Cavalcanti NB \& Resende GM (2007) Consumo de xiquexique (Pilocereus gounellei (A. Weber ex K. Schum.) Bly. ex Rowl) por caprinos no semi-árido da Bahia. Revista Caatinga 20: 22-27.

Chaves EMF, Barros RFM \& Araújo FS (2007) Flora Apícola do Carrasco no Município de Cocal, Piauí, Brasil. Revista Brasileira de Biociências 5: 555-557.

Chytrý M, Tichý L, Holt J \& Botta-Dukát Z (2002) Determination of diagnostic species with statistical fidelity measures. Journal of Vegetation Science 13: 79-90. 
Conceição AA, Giulietti AM \& Meirelles ST (2007a) Ilhas de vegetação em afloramentos de quartzito-arenito no Morro do Pai Inácio, Chapada Diamantina, Bahia, Brasil. Acta Botanica Brasilica 21: 335-347.

Conceição AA, Pirani JR \& Meirelles ST (2007b) Floristics, structure and soil of insular vegetation in four quartzite-sandstone outcrops of Chapada Diamantina", Northeast Brazil. Brazilian Journal of Botany 30: 641-656.

Cornejo X \& Iltis HH (2008). A revision of the American species of the genus Crateva (Capparaceae). Harvard Papers in Botany 13: 121-135.

Costa ECS, Lopes SF \& Melo JIM (2015) Floristic similarity and dispersal syndromes in a rocky outcrop in semi-arid Northeastern Brazil. Revista de Biología Tropical 63: 827-843.

Costa RC, Araújo FS \& Lima-Verde LW (2007) Flora and life-form spectrum in an area of deciduous thorn woodland (caatinga) in northeastern, Brazil. Journal of Arid Environments 68: 237-247.

Cunha TJF, Petrere VG, Silva DJ, Mendes AMS, Melo RF, Oliveira Neto MB, Silva MSL \& Alvárez IA (2010) Principais solos do semiárido tropical brasileiro: caracterização, potencialidades, limitações, fertilidade e manejo. In: Sá IB \& Silva PCG (eds.) Semiárido brasileiro. Pesquisa, desenvolvimento e inovação. Embrapa Semiárido, Petrolina. Pp. 49-87.

Destro N, Szatmari P \& Ladeira EA (1994) Post-Devonian transpressional reactivation of a Proterozoic ductile shear zone in Ceará, NE Brazil. Journal of Structural Geology 16: 35-45.

Ferraz EMN, Rodal MJN, Sampaio EVSB \& Pereira RCA (1998) Composição florística em trechos de vegetação de caatinga e brejo de altitude na região do Vale do Pajeú, Pernambuco. Revista Brasileira de Botânica 21: 7-15.

Figueiredo MA (1997) A cobertura vegetal do Ceará (Unidades Fitoecológicas). In: Atlas do Ceará. Governo do estado do Ceará, IPLANCE, Fortaleza, $65 \mathrm{p}$.

Figueiredo JM (2010) Revegetação de áreas antropizadas da Caatinga com espécies nativas. Tese de Mestrado. Universidade Federal de Campina Grande, PatosPB. $47 \mathrm{p}$.

Forzza RC (2001) Filogenia da tribo Puyeae Wittm. e revisão taxonômica do gênero Encholirium Mart. ex Schullt. \& Schullt. F. (Pitcairnioideae Bromeliaaceae). Tese de Doutorado. Universidade de São Paulo, São Paulo. 208p.

Forzza RC (2005) Revisão taxonómica de Encholirium Mart. (Pitcairnioideae - Bromeliaceae). Boletim de Botânica da Universidade de São Paulo 23: 1-49.

Forzza RC, Christianini AV, Wanderley MGL \& Buzato S (2003) Encholirium (Pitcairnioideae - Bromeliaceae): conhecimento atual e sugestões para conservação. Vidália 1: 7-20.

França F, Melo E \& Santos CC (1997) Flora de inselbergs da região de Milagres, Bahia, Brasil: I. Caracterização da vegetação e lista de espécies de dois inselbergs. Sutientibus 17: 163-184.

França F, Melo E, Santos AKA, Melo JGAN, Marques M, Silva-Filho MFB, Moraes L \& Machado C (2005) Estudos ecológico e florístico em ilhas de vegetação de um inselberg no semi-árido da Bahia, Brasil. Hoehnea 32: 93-101.

Freise FW (1938) The drought region of northeastern Brazil. Geographical Review 28: 363-378.

Géhu JM \& Rivas-Martínez S (1981) Notions fondamentales de phytosociologie. In: Dierschke $\mathrm{H}$ (ed.) Syntaxonomie. Cramer, Vaduz. Pp 5-33.

Gomes P \& Alves M (2009) Floristic and vegetational aspects of an inselberg in the semi-arid region of Northeast Brazil. Edinburgh Journal of Botany 66: 329-346.

Gomes P \& Alves M (2010) Floristic diversity of two crystalline rocky outcrops in the Brazilian northeast semi-arid region. Brazilian Journal of Botany 33: 661-676.

Gomes P, Costa KCC, Rodal MJN \& Alves M (2011) Checklist of Angiosperms from the Pedra Furada Municipal Park, northeastern Brazil.Check List 7: 173-181.

Gomes P \& Sobral-Leite M (2013) Crystalline rock outcrops in the Atlantic forest of northeastern Brazil: vascular flora, biological spectrum, and invasive species. Brazilian Journal of Botany 36: 111-123.

Gomes RP (1977) Forragens fartas na seca. 4. ${ }^{\mathrm{a}}$ ed. Nobel, São Paulo. 233p.

Hill MO (1979) TWINSPAN: a FORTRAN program for arranging multivariate data on an ordered twoway table by classification of the individuals and attributes. Cornell University, New York. 90p.

Hintze J \& Nelson R (1998) Violin Plots: a box plotdensity trace synergism. The American Statistician 52: 181-184.

IBGE - Instituto Brasileiro de Geografia e Estatística (2004) Mapa de biomas do Brasil: primeira aproximação. Disponível em $<$ http://www.ibge.gov. br/home/presidencia/noticias/21052004biomashtml. shtm>. Acesso em 15 abril 2016.

IBGE - Instituto Brasileiro de Geografia e Estatística (2012) Manual técnico da vegetação brasileira (manuais técnicos de geociências n ${ }^{\circ}$. 1). 2. ${ }^{a}$ ed. IBGE, Rio de Janeiro. 271p.

Kluge M \& Brulfert J (2000) Ecophysiology of vascular plants on inselbergs. In: Porembski S \& Barthlott W (eds.) Inselbergs: biotic diversity of isoleted rock outcrops in tropical and temperate regions. SpringerVerlag, Berlin. Pp. 143-176.

Larson DW, Matthes U \& Kelly PE (2000) Cliff ecology: pattern and process in cliff ecosystems. Cambridge University Press, Cambridge. 360p.

Leal IR, Silva JMC, Tabarelli M \& Lacher TE (2005) Changing the course of biodiversity conservation in the Caatinga of northeastern Brazil. Conservation Biology 19: 701-706. 
Loiola MIB, Rocha EA, Baracho GS \& Agra MF (2009) Flora da Paraíba, Brasil: Combretaceae. Acta Botânica Brasílica 23: 330-342.

Lorenzi H (2008) Árvores brasileiras: manual de identificação e cultivo de plantas arbóreas nativas do Brasil. 5. ${ }^{\text {a }}$ ed. Instituto Plantarium, Nova Odessa. $1384 \mathrm{p}$.

Maia GN (2004) Caatinga: árvores e arbustos e suas utilidades. D\&Z Computação Gráfica e Editora, São Paulo. 413p.

Marques FA, Nascimento AF, Araújo Filho JC \& Silva AB (2014) Solos do Nordeste. EMBRAPA, Brasília. 8p.

Martinelli G (2007) Mountain biodiversity in Brazil. Brazilian Journal of Botany 30: 587-597.

Martins FR \& Batalha MA (2011) Formas de vida, espectro biológico de Raunkiaer e fisionomia da vegetação. In: Felfili JM, Eisenlohr PV, Fiuza de Melo MMR, Andrade LA \& Meira Neto JAA (org.) Fitossociologia no Brasil: métodos e estudos de caso. Vol. 1. Editora UFV, Viçosa. Pp. 44-85.

Meirelles ST, Pivello VR \& Joly CA (1999) The vegetation of granite rock outcrops in Rio de Janeiro, Brazil, and the need for its protection. Environmental Conservation 26: 10-20.

Moraes AO, Melo E, Agra MF \& França FA (2009) Família Solanaceae nos "Inselbergs" do semi-árido da Bahia, Brasil. Iheringia 64: 109-122.

Moro MF, Lughadha EN, Araújo FS \& Martins FR (2016) A phytogeographical metaanalysis of the semiarid Caatinga domain in Brazil. The Botanical Review 82: 91-148.

Moro MF, Lughadha EN, Filer DL, Araújo FS \& Martins FR (2014) A catalogue of the vascular plants of the Caatinga phytogeographical domain: a synthesis of floristic and phytosociological surveys. Phytotaxa 160: 1-118.

Moro MF, Macedo MB, Moura-Fé MM, Castro ASF \& Costa RC (2015a) Vegetação, unidades fitoecológicas e diversidade paisagística do estado do Ceará. Rodriguésia 66: 717-743.

Moro MF, Silva IA, Araújo FS, Lughadha EN, Meagher TR \& Martins FR (2015b) The role of edaphic environment and climate in structuring phylogenetic pattern in seasonally dry tropical plant communities. PLoS ONE 10: e0119166.

Moro MF, Sousa VC, Oliveira-Filho AT, Queiroz LP, Fraga CN, Rodal MJN, Araujo FS \& Martins FR (2012) Alienígenas na sala: o que fazer com espécies exóticas em trabalhos de taxonomia, floristica e fitossociologia? Acta Botanica Brasílica 26: 991-999.

Morrone JJ (2001) Biogeografía de América Latina y el Caribe. M\&T - Manuales y Tesis SEA, vol. 3. Zaragoza (España). 55p.

Neves BBDB (1998) The Cambro-Ordovician of the Borborema province. Boletim IG-USP, Série Científica 29: 175-193.
Oliveira G, Araújo MB, Rangel TF, Alagador D \& DinizFilho JAF (2012) Conserving the Brazilian semiarid (Caatinga) biome under climate change. Biodiversity and Conservation 21: 2913-2926.

Pereira MMD, Braga PET \& Guiomar N (2014) Análise dos diferentes estágios de desenvolvimento da caatinga em Sobral, Ceará, Brasil. Revista da Casa da Geografia de Sobral 16: 46-65.

Pessoa E \& Alves M (2014) Orchidaceae em afloramentos rochosos do estado de Pernambuco, Brasil. Rodriguésia 65: 717-734.

Peulvast J-P \& Betard F (2015) Landforms and landscape evolution of the equatorial margin of Northeast Brazil: an overview. Springer, Cham. 186p.

Porembski S (2007) Tropical inselbergs: habitat types, adaptive strategies and diversity patterns. Brazilian Journal of Botany 30: 579-586.

Porembski S, Barthlott W, Dörrstock S \& Biedinger N (1994) Vegetation of rock outcrops in Guinea: granite inselbergs, sandstone table mountains and ferricretes - remarks on species numbers and endemism. Flora 189: 315-326.

Porembski S, Martinelli G, Ohlemuller R \& Barthlott W (1998) Diversity and ecology of saxicolous vegetation mats on inselbergs in Brazilian Atlantic forest. Diversity and Distributions 4: 107-119.

Porembski S, Szarzynski J, Mund J-P \& Barthlott W (1996) Biodiversity and vegetation of small-sized inselbergs in a West African rain forest (Taï, Ivory Coast). Journal of Biogeography 23: 47-55.

Porto PAF, Almeida A, Pessoa WJ, Felix LP \& Trovão D (2008) Composição florística de um inselbergue no Agreste paraibano, município de Esperança, Nordeste do Brasil. Revista Caatinga 21: 214.

Prata APN, Amaral MCE, Farias MCV \& Alves M (2013) Flora de Sergipe. Gráfica e Editora Triunfo, Aracaju. 8p.

Queiroz LP (2009) Leguminosas da Caatinga. Multimídia Indústria, Comércio e Editora, Feira de Santana. 443p.

R Development Core Team, 2015. R: a language and environment for statistical computing. $\mathrm{R}$ foundation for statistical computing. Vienna, Austria. Disponível em <https://www.r-project. org/>. Acesso em 15 novembro 2017.

Ramalho M, Batista MA \& Silva M (2004) Xylocopa (Monoxylocopa) abbreviata Hurd \& Moure (Hymenoptera: Apidae) e Encholirium spectabile (Bromeliaceae): uma associação estreita no semiárido do Brasil Tropical. Neotropical Entomology 33: 417-425.

Raunkiaer C (1934). The life forms of plants and statistical geography. Claredon, Oxford. 104p.

Ribeiro KT, Medina BMO \& Scarano FR (2007). Species composition and biogeographic relations of the rock outcrop flora on the high plateau of Itatiaia, SE-Brazil. Revista Brasileira de Botânica 30: 623-639. 
Robertson AW, Kirshner S \& Smyth P (2004) Downscaling of daily rainfall occurrence over northeast Brazil using a hidden Markov model. Journal of Climate 17: 4407-4424.

Rodal MJN, Sampaio EVSB \& Figueiredo MA (org.) (2013) Manual sobre métodos de estudos florístico e fitossociológico - ecossistema caatinga. Sociedade Botânica do Brasil, Brasília. 24p.

Rodrigues JMD \& Lima EC (2015) Análise dos sistemas ambientais da sub-bacia hidrográfica do rio Bom Jesus-Taperuaba-CE. Revista da Casa da Geografia de Sobral 17: 60-79.

Rodrigues WA \& Pires JM (1988) Inventário fitossociológico. Anais do Encontro sobre Inventários Florísticos na Amazônia, Manaus. 5p.

Sales-Rodrigues J, Brasileiro JCB \& Melo JIM (2014) Flora de um inselberg na mesorregião agreste do estado da Paraíba-Brasil. Polibotânica 37: 47-61.

Santos CAC, Brito JIB, Rao TVR \& Menezes HEA(2009) Tendências dos índices de precipitação no estado do Ceará. Revista Brasileira de Meteorologia 24: 39-47.

Scarano FR (2007) Rock outcrop vegetation in Brazil: a brief overview. Brazilian Journal of Botany 30 : 561-568.

Silva FKG, Lopes SF, Lopez LCS, Melo JIM \& Trovão DMBM (2014) Patterns of species richness and conservation in the Caatinga along elevational gradients in a semiarid ecosystem. Journal of Arid Environments 110: 47-52.

Silva JGM, Silva DS, Pereira WE, Diniz MCNM, Silva GJAM \& Medeiros MR (2011) Características morfológicas e produção do xiquexique cultivado em diferentes densidades. Revista Centauro 2: 8-17.

Silva JGM, Silva DS, Ferreira MA, Lima GFC, Melo AAS \& Diniz MCNM (2005) Xiquexique [Pilosocereus gounellei (A. Weber ex K. Schum.) Bly. ex Rowl.] em substituição à silagem de sorgo (Sorghum bicolor L. Moench) na alimentação de vacas leiteiras. Revista Brasileira de Zootecnia 34: 1408-1417.

Silva KB, Alves EU, Bruno RLA, Gonçalves EP, França PRC, Nascimento IL \& Lima CR (2007) Substratos para germinação e vigor em sementes de Crataeva tapia L. Revista Brasileira de Biociências 5: 111-113.

Silva TDS, Felix LP \& Melo JIMD (2015) Bromeliaceae and Orchidaceae on rocky outcrops in the Agreste Mesoregion of the Paraíba State, Brazil. Hoehnea 42: 345-365.

Smith LB \& Downs RJ (1974) Flora neotropica Monograph 14 (Pitcairnioideae) (Bromeliaceae). Hafner Press, New York. 658p.

Soares-Neto RL, Magalhães FAL, Tabosa FRS, Moro MF, Costa e Silva MB \& Loiola MIB (2014) Flora of Ceará state, Brazil: Capparaceae. Rodriguésia 65: 671-684.

Sobrinho JF (2009) Geo-história ambiental do Vale do Acaraú. Edições universitárias, Universidade Estadual Vale do Acaraú. 97p.

Sokal RR \& Rohlf FJ (1995) Biometry: the principles and practice of statistics in biological research. $3^{\text {rd }}$ edition. W. H. Freeman and Co. New York. 880p.

Sun L, Li H, Ward MN \& Moncunill DF (2007) Climate variability and corn yields in semiarid Ceará, Brazil. Journal of Applied Meteorology and Climatology 46: 226-240.

Tabarelli M, Vicente A \& Barbosa DCA (2003) Variation of seed dispersal spectrum of woody plants across a rainfall gradient in north-eastern Brazil. Journal of Arid Environments 53: 197-210.

Taylor NP \& Zappi DC (2002) Distribuição das espécies de Cactaceae na caatinga. In: Sampaio EVSB, Giulietti AM, Virgínio J \& Gamarra-Rojas CFL (eds.) Vegetação e flora da caatinga. APNE/CNIP, Recife. Pp.123-125.

Tichý L (2002) JUICE, software for vegetation classification. Journal of Vegetation Science 13: 451-453.

Tichý L \& Chytrý M (2006) Statistical determination of diagnostic species for site groups of unequal size. Journal of Vegetation Science. 17: 809-818.

Tölke E, Silva JB, Pereira ARL \& Melo JIM (2011) Flora vascular de um inselbergue no estado da Paraíba, Nordeste do Brasil. Biotemas 24: 39-48.

Werner PC \& Gerstengarbe FW (2003) The climate of Piauí and Ceará. In: Gaiser T, Krol MS, Frischkorn H \& Araújo JC (eds.) Global change and regional impacts. Water availability and vulnerability of ecosystems and society in the semiarid northeast of Brazil. Springer, Berlin, Heidelberg. Pp. 81-85.

Wickham H (2016) ggplot2: elegant graphics for data analysis. Springer, New York. 260p. 